(Aus der Universitäts-Frauenklinik in Zürich.)

\title{
Zur Frage der künstlichen Unterbrechung der Schwangerschaft wegen Lungentuberculose.
}

Von

Dr. C. Pradella,

in Basel, früher in Davos.

Auf Grund der Bearbeitung der ziemlich umfangreichen einschlägigen Literatur ${ }^{1}$ ) und der Verwendung eines Theiles des durch die Güte des Herrn Professor Wyder uns zugänglich gemachten diesbezüglichen Materiales der Züricher Universitäts-Frauenklinik sind wir über den Stand der Streitfrage zu einer Reihe von Erfahrungssätzen und Schlussfolgerungen gelangt, welche in einer im Jahre 1906 erschienenen Dissertation (Zürich) ${ }^{2}$ ) niedergelegt sind. Indem wir die wiehtigsten derselben an dieser Stelle wiederholen, verweisen wir einzelner Angaben über das Material der Literatır wegen auf die genannte Abhandlung.

Wir werden weiter unten über die Fälle der Züricher Universitäts-Frauenklinik berichten und es soll der Versuch, dem in der Praxis stehenden Arzte für seine Entscheidung und sein Handeln eine Wegleitung zu geben, den Abschluss auch vorliegender Arbeit bilden.

Die Betrachtungen, Erfahrungssätze und Schlussfolgerungen, zu welchen wir, die Ergebnisse der Literatur und unseres eigenen Beitrages zusammenfassend, gelangten, sind folgende:

1) Mit Mauriceaux (1645) beginnend war Kellmann's Dissertation (Juli 1905) die letzte Abhandlung, welche wir, um zu einem Abschluss zu gelangen, für unsere Arbeit benützt haben

2) C. Pradella. Zur Frage der künstlichen Unterbrechung der Schwangerschaft wegen Lungentuberculose. Inaugural-Dissertation, Zürich, 1906. 
Die seit Jahrhunderten beobachteten Wechselbeziehungen zwischen beiden führte zur Erkenntniss, dass die Generationsvorgänge (Schwangerschaft, Geburt und Wochenbett) ein wichtiges Glied in der Reihe der ätiologischen Momente der Lungentuberculose bilden.

Es ist erwiesen, dass dieselben in manchen Fällen auf die Lungentuberculose in keiner Weise einen Einfluss ausüben.

Es seht ferner fest, dass bei ein und demselben Individuum einmal im Verlaufe der Generationsvorgänge die Krankheit beeinflusst wird, ein andermal dieselbe unbeeinflusst blejbt. Dieser wechselnde Einfluss macht sich auch geltend innerhalb der einzelnen Phasen ein und derselben Generationsperiode.

Für einen günstigen Einfluss der Generationsvorgänge sind ausser einer stattlichen Anzahl von Aerzten älterer Schule in neuerer Zeit unter Anderen Wernich, Ruehle und H. W. Freund eingetreten und zwar diese drei Genannten speciell für eine günstige Beeinflussung durch die Gravidität. Wernich bekennt sich völlig zu der alten Meinung, dass chronische pneumonische Processe in der Gravidität milder verlaufen und geradezu den Glauben an eine gewisse Latenz erwecken.

Ruehle äussert sich darüber folgendermaassen: "Während die Gravidität meist Stillstand bewirkt, bewirkt das Wochenbett einen floriden Verlauf und fübrt zuweilen in wenigen Monaten zum Tode."

H. W. Freund, auf Wernich Bezug nehmend, sagt: „Wernich hält es sogar mit der alten Anschaung von dem günstigen Einfluss der Gravidität, entschieden für viele Fälle nicht mit Unrecht."

Wir halten uns nicht für berechtigt die Richtigkeit der Beobachtungen und Erfahrungen der diesen Standpunkt vertretenden Aerzte anzuzweifeln, um so weniger, als wir selbst mehrfach Gelegenheit hatten, während der Schwangerschaft bei lungentuberculösen Frauen ganz erhebliche Besserung, sogar Stillstand der tuberculösen Processe zu beobachten. Allerdings glaubten wir bei unseren Kranken diesen Erfolg nicht dem Einflusse der Gravidität, sondern der antituberculösen Behandlung zuschreiben zu müssen und der weitere Verlauf hat uns darin bestärkt. Damit soll nicht in Abrede gestellt werden, dass bei manchen Patientinnen, bei welchen eine Ein- und Mitwirkung anderer günstiger Factoren auszuschliessen ist, die Krankheit durch die Schwangerschaft günstig beeinflusst werden könne. 
Wenn nun auch alle angeführten Modificationen in den Wechselbeziehungen zwischen Generationsworgängen und Lungentuberculose beobachtet sind, so steht heute doch unumstösslich fest: Der A usbruch, der ungünstige Verlauf und Ausgang der Lungentuberculose bei einer grossen Anzahl von Frauen in jeder Phase der Generationsvorgänge, sind auf diese zurückzuführen.

Eine weitgehende Sympathie oder Solidarität zwischen Lungen und Uterus, ein gewisser Antagonismus zwischen Schwangersehaft und Lungentuberculose, eine ableitende Wirkung der Gravidität auf diese Krankheit, ein Zustand von Herzhypertrophie, wie er bei schwangeren Frauen bestehe, ein sogenannter "Rachitisme", der während der Schwangerschaft vorkomme, wurden zur Erklärung des günstigen Einflusses herangezogen.

Die gewaltigen Veränderungen im Bereiche des Kreislaufsund Athmungsapparates, wie sie durch die Gravidität physiologischerweise bedingt und, wenigstens theilweise, schon lange bekannt waren, sowie deren Rückwirkung auf den Gesammtorganismus und die einzelnen Organe, insbesondere auf Herz und Lungen der tuberculösen Frauen, wurden und werden verantwortlich gemacht für den schädlichen Einfluss der Schwangerschaft, andrerseits die plötzliche Aenderung und der Ausgleich dieses Zustandes nach der Entbindung für die verderbliche Einwirkung der Geburt, des Wochenbettes und der Folgezeit. Auch der mit dem Geburtsact verbundene Traumatismus wird als Ursache des schlimmen Verlaufes angeschuldigt. Wir übergehen andere schon als pathologisch anzusprechende $\mathrm{Zu}$ stände, welche die Verschlimmerung herbeiführen sollen und erwähnen nur noch Hanau's Erklärungsversuch. Er erklärt im Anschluss an vier nach der Geburt rapid letal verlaufende Fälle von bestehender alter Phthise an der Hand des Sectionsbefundes den verderblichen Einfluss der Geburt besonders mit Rücksicht auf die anatomische Form der frischen Herde einzig und allein (in seinen Fällen) als Folge einer Autoinfection durch Aspiration von Cavernenmassen, zu welcher während der Geburt durch die heftigen Respirationsanstrengungen hinreichend Gelegenheit geboten sei.

Die meisten Berichte über die Häufigkeit der schädlichen Wirkung der Generationsvorgänge bringen nur unbestimmte Ausdrücke. Um so werthvoller sind die Angaben einzelner Autoren, welche Zahlenverhältnisse angeben. 
Jakubasch (z. n. Kellmann) bringt in 24 pCt. der Fälle die Entstehung der Tuberculose mit den Generationsvorgängen in Verbindung.

Jacob und Pannwitz führen bei 337 phthisischen Frauen 84 Mal die Entstehung oder Verschlimmerung der Tuberculose auf Gravidität, Geburt und Wochenbett zurück, also bei 25 pCt. der Kranken.

Turban sah in unmittelbarem Anschluss an Gravidität, Wochenbett und Stillen den ersten Ausbruch der Lungentuberculose in 18 Fällen. Unter den Patienten weiblichen Geschlechtes der Anstalt betanden sich 62 Frauen, woyon eine als kinderlos verheirathet bezeichnet ist. Nimmt man an, dass alle anderen Frauen geboren haben, so wäre der erste Ausbruch der Krankheit in Folge der Generationsvorgänge in 29 pCt. der Fälle erfolgt und zwar bei Frauen in guter bis vorzüglicher Lebensstellung.

Flint (z. n. Towsend) beobachtete 87 phthisische Patientinnen; $22 \mathrm{mal}$, also bei $25 \mathrm{pCt}$. derselben, erfolgte die Erkrankung wegen Conception und zwar 10 mal ( 45 pCt.) während der Schwangerschaft, $12 \mathrm{mal}$ ( $55 \mathrm{pCt}$.) nach der Entbindung.

Merletti hat eine Verschlimmerung der Lungentuberculose durch die erwähnten Vorgänge in $50 \mathrm{pCt}$. der Krankheitsfälle beobachtet.

Maragliano giebt folgende Daten: von 385 an ausgesprochener Phthise leidenden Frauen war 226 mal, das heisst bei $59 \mathrm{pCt}$. derselben der Beginn der Erkrankung auf eine Geburt oder Schwangerschaft zurückzuführen.

Deibel's Gesammtmaterial von 50 Fällen ergiebt eine Verschlechterung im Verlaufe der Gravidität in 64 pCt. der Krankheitsfälle.

Kaminer fand Verschlimmerung bei 66 pCt., keinen Einfluss bei $16 \mathrm{pCt}$. seiner Kranken; bei 18 pCt. war eine Beeinflussung nicht sicher nachweisbar.

Die Zusammenstellung von Eich ergiebt folgende Resultate: 75 pCt. der Fälle wurden in der Gravidität oder im Puerperium progredient und zwar erfolgte in 64 pCt. die Verschlechterung in ersterer, in $11 \mathrm{pCt}$. in letzterem. In $25 \mathrm{pCt}$. derselben war ein ungünstiger Einfluss überhaupt nicht nachweisbar. Es handelte sich bei diesen 20 Frauen um Fälle von ausgeheilter Phthise.

Lebert gelangt zu folgendem Schlusse: Gravidität und 
Wochenbett wirken in $3 / 4$ der Fälle nachtheilig in Bezug auf Entwicklung und rasches Fortschreiten der Lungentuberculose.

Das aus der Literatur von uns zusammengestellte Material umfasst: 23 spontane Aborte, 76 spontane Frühgeburten, 814 spontane rechtzeitige Geburten, 62 künstliche Fehl- und 60 ebensolcho Frühgeburten, im Ganzen 1035 Fälle.

Bevor wir über einige Resultate desselben berichten, wollen wir ausführen, in welcher Weise dieses Material ron uns gesammelt und verarbeitet wurde. Wir haben fast alle erreichbaren Fälle sowohl spontanen Geburtsverlaufes als künstlicher Entbindung aufgenommen und eine Anzahl derselben, soweit dies angängig war und der Mühe lohnte, nach den bei unserm eigenen Material berücksichtigten Gesichtspunkten zusammengestellt. Ueber Beruf, hereditäre Belastung, Zahl der Schwangersehaften, Stadium der Gravidität, Complicationen, Operationsmethode, Verlauf der Geburt, waren die Angaben so spärlich und lückenhaft, dass wir diese Rubriken nicht berücksichtigt haben. Für die Endresultate wurden nur solche Fälle verwendet, bei welchen nicht nur über den Verlauf kurz nach der Geburt beziehungsweise im Wochenbett, sondern auch über den weitern Verlauf und Ausgang im Anschluss an die Entbindung ein Bericht vorhanden war. Wir bezeichneten als positiven Erfolg eine im Anschluss an die Entbindung, sei dieselbe eine spontane oder künstliche, erfolgte andauernde Besserung - bei Fällen, in welchen während der Gravidität eine Verschlimmerung nicht eintrat, ein Stationärbleiben - Stillstand, beziehungsweise Heilung der Lungentuberculose mit Berücksichtigung, wo solche Angaben vorhanden waren, der Wiedererlangung oder Erhaltung der Arbeits- oder Leistungsfähigkeit, mit negativem Erfolg eine nach der Geburt andauende - bei während der Schwangerschaft stationär bleibenden Fällen eine nach der Entbindung auftretende - Verschlimmerung, welche schlimmsten Falles zum Tode, in Fällen, wo es schliesslich doch zum Stillstand und zur Ausheilung kommt, zur Verschlechterung des Krankheitsgrades geführt hat. Auch hier berücksichtigten wir die Arbeits- oder Leistungsfähigkeit der Kranken, wollen aber gleich beifügen, dass Arbeits- oder Leistungsfähigkeit einerseits und Stillstand oder Heilung andrerseits keineswegs sich deckende Begriffe sind.

Es mögen hier noch einige weitere Bemerkungen über das zur Lösung der Streitfrage dienliche Material Platz finden. Wie angedeutet sind die Literaturberichte vielfach sehr lückenhaft, nament- 
lich auch über das Krankheitsstadium und den weitern Verlauf und Ausgang der Krankheit nach erfolgter Entbindung und in der Folgezeit, soweit ein Zusammenhang zwischen beiden in Frage kommt.

Wir hätten viel mehr Fälle zur Penutzung heranziehen können, wenn wir einen andern Maassstab anlegten, glauben aber, es gereiche der Arbeit eher zum Nutzen als zum Vorwurf, wenn wir die Auswahl derselben, wo immer möglich, nach denselben Gesichtspunkten trafen, nach welchen wir unser eigenes Material verarbeitet haben, auf die Gefahr hin manche Fälle zu verlieren.

Es muss viel mehr als bisher darauf hingearbeitet werden, das einschlägige Material nach einheitlichen Gesichtspunkten zu beobachten, zu sammeln und zu verwerthen.

Neben Material aus der minder- und unhemittelten Klasse der Bevölkerung müssen die Erfahrungen aus der Praxis gut und bestsituirter Kranken zur Kenntniss gebracht werden. Es sind Krankheitsfälle aller Stadien in dasselbe aufzunehmen. Man scheint, wenige Ausnahmen abgerechnet, keine Veranlassung zu finden, die Beobachtungen und Resultate leichter Fälle und günstigen Verlaufes zu veröffentlichen. Ganz besonders trifft dies $z u$ bei den Spontangeburten.

Namentlich aber ist dringend nothwendig, dass die Patientinnen und zwar nicht nur nach künstlichen, sondern namentlich auch nach spontanen Entbindungen solange beobachtet werden, bis ein abschliessendes Urtheil über den endgiltigen Ausgang der Krankheit möglich ist.

Auch die lungentuberculösen Wöchnerinnen müssen aus den klinischen Anstalten nach der im Allgemeinen üblichen Aufenthaltsdauer entlassen werden. Manche werden auf interne Abtheilungen transferirt, andere begeben sich in häusliche, private oder anderweitige Anstaltspflege. Aufgabe der Aerzte, in deren Hände die Behandlung der tuberculösen Wöchnerinnen gelegt ist oder wird, muss es sein, den Einfluss der Geburt und des Wochenbettes und all' der übrigen mit in Betracht kommenden Factoren auf die Krankheit zu beobachten und zwar, um es noch einmal zu sagen, solange, bis ein endgiltiges Urtheil über den definitiven Ausgang der Lungentuberculose ermöglicht ist. Eine solche Beobachtung und Controle wird ja zweifellos nicht allgemein, aber bei gutem Willen in vielen Fällen durchführbar sein. Wie schwer es hält, nachträglich erst zuverlässige Angaben und Berichte zu erlangen, 
davon konnten wir uns beim Material der Züricher Klinik überzeugen.

Um auf die Häufigkeit der verderblichen Einwirkung der Schwangerschaft auf die Lungentuberculose zurückzukommen, so geht aus unserm Literatur-Material hervor, dass, alle Krankheitsgrade und Fälle inbegriffen, eine solche in $95 \mathrm{pCt}$. derselben vorhanden war.

Zur Erklärung dieses auffallend hohen Procentsatzes sei beigefügt, dass, soweit in diesem Sinne verwendbare Aufzeichnungen in den Krankengeschichten sich vorfanden, dieselben darauf hinwiesen, dass bei der Grosszahl der in Frage stehenden Patientinnen es sich um in sehr ungünstigen Lebensverhältnissen befindliche Frauen handelte.

Ueber etwa $6 / 10$ der Literatur-Fälle fehlen Angaben hinsichtlich des Krankheitsgrades, von den übrigen $4 / 10$ stand der vierte Theil der Frauen bestimmten Berichten zufolge im ersten Stadium der Lungentuberculose, die restirenden $3 / 4$ vertheilen sich über alle Stadien, so dass auch von diesen eine Anzahl als leicht erkrankt gelten darf.

Eich, welcher über annähernd $6 / 10$ Fälle von Phthisis incipiens verfügt, berechnet trotz der Mehrzahl der leichten Erkrankungen den Procentsatz der durch die Gravidität ungünstig beeinflussten Fälle auf 75 pCt., Kaminer auf 66 pCt. bei einem Material, wo die Hälfte der Frauen leicht krank war. Auch aus anderen Berichten geht hervor, dass eine verhältnissmässig grosse Zahl der durch die Schwangerschaft verderblich beeinflussten Fälle zu den leichten gehörten.

Wir fügen daher dem Erfahrungssatze vom ungünstigen Einflusse speciell der Gravidität auf diese Krankheit als Zusatz bei: Der ungünstige Einfluss, welchen die Schwangersehaft auf die Lungentuberculose ausübt, äussert sich ohne Rücksicht auf das Stadium der Erkrankung.

Die oben angeführten Zahlenbeispiele dürften geeignet sein, die lange bestehende Legende, die Gravidität verleihe gegen Erkrankung an Lungentuberculose Immunität, zu zerstören.

Von den erwähnten 1035 graviden lungentuberculösen Frauen sind nur 13 oder $1 \frac{1}{4}$ pCt. unentbunden im Verlaufe der Schwangerschaft gestorben, ein Ergebniss, das die längst gemachte Beobachtung bestätigt: Ein tödtlicher Ausgang im Verlaufe der 
Gravidität bei Lungentuberculose ist ein seltenes Ereigniss.

Ueber den Einfluss der Geburt und des Wochenbettes auf die Lungentuberculose gab das Material der Literatur uns folgende Aufschlüsse :

Im Puerperium erfolgte nach der spontanen Entbindung in etwa $3 / 5$ der Fälle eine Verschlimmerung, in je annähernd $1 / 5$ war ein Einfluss nicht zu bemerken, beziehungsweise eine Besserung eingetreten. Das Zahlenverhältniss von $3 / 5$ ungünstig zu $2 / 5$ günstig verlaufender und unbeeinflusst gebliebener Fälle dürfte dem thatsächlichen Verhältnisse der schwerer und leichter Erkrankten nahe kommen oder entsprechen.

Auch der günstige Wochenbettverlauf bei den künstlichen $\mathrm{Ab}$ orten $(83 \mathrm{pCt}$. Besserung, $7 \mathrm{pCt}$. Verschlimmerung, $10 \mathrm{pCt}$. ohne Beeinflussung), der ungünstige der künstlichen Frühgeburten (8 pCt. Besserung, 42 pCt. Verschlimmerung, 50 pCt. ohne Beeinflussung) erklärte sich wohl aus dem Umstande, dass bei den ersteren es sich grösstentheils um leichte Erkrankung, bei den letzteren fast ausschliesslich um schwerkranke Frauen handelte.

Da indessen bei vielen Wöchnerinnen aller Stadien sowohl nach spontaner als künstlicher Entbindung im Puerperium auf eine vorübergehende Besserung Verschlimmerung erfolgte und umgekehrt, so darf das Resultat des Wochenbettverlaufes nur in denjenigen Fällen als endgüliges angesehen werden, in denen es als solches in unserem Sinne schon innerhalb des Verlaufes des Puerperiums festzustellen ist.

Hatte uns der Verlauf im Wochenbett auf einen Zusammenhang zwischen diesem und dem Krankheitsstadium hingewiesen, so zeigte derselbe sich in unverkennbarer Weise, als wir den weiteren Verlauf und endgültigen Ausgang der Krankheit in Betracht zogen.

Bei den spontanen Entbindungen fehlen meist Berichte über die Endresultate. Soweit Angaben über den Krankheitsgrad vorhanden waren, liess sich feststellen, dass bei spontanen, sowohl vorzeitigen als zeitigen Entbindungen der Verlauf und Ausgang um so günstiger oder ungünstiger sich gestaltete, je leichter oder schwerer die Erkrankung war. Die Verschiebungen, die sich in den Zahlenverhältnissen ergaben, je nachdem das Stadium bei der Betrachtung der Endresultate berücksichtigt wurde oder nicht, fielen uns am meisten auf beim Material der spontanen Geburten Kaminer's. 
Kaminer bediente sich der Turb'an'schen Stadieneintheilung, ohne indess für die Beurtheilung des Ausganges hieraus die möglichen Consequenzen zu ziehen.

Seine Statistik umfasst 50 Fälle. Nach Abzug von 2 spontanen Aborten, 15 künstlichen Frühgeburten und von 6 Fällen, in welchen die betreffenden Frauen noch nicht geboren hatten, verbleiben 27 spontane zeitige Geburten. Von 23 dieser Frauen ist der weitere Verlauf und Ausgang in unserem Sinne bekannt.

Die Resultate ohne Berücksichtigung des Krankheitsgrades waren folgende: Es wurden in der Folge arbeitsfähig $8=35 \mathrm{pCt}$. der Kranken, nicht arbeitsfähig blieben $3=13$ pCt., gestorben sind $12=52$ pCt., davon 7 schon im Wochenbett.

Wird das Krankheitsstadium in Betracht gezogen, so resultiren folgende Zahlen: Von 8 Frauen des ersten Stadiums wurden arbeitsfähig $7=871 / 2 \mathrm{pCt}$., nicht arbeitsfähig blieb $1=12^{1} / 2 \mathrm{pCt}$. Bei 4. Frauen zweiten Stadiums war das Endresultat: Arbeitsfähigkeit bei $1=25 \mathrm{pCt}$., Arbeitsunfähigkeit bei $2=50 \mathrm{pCt}$., tödtlicher Ausgang bei $1=25 \mathrm{pCt}$. Von den 11 Frauen des dritten Stadiums gingen alle zu Grunde.

Die Zusammenstellung der oben erwähnten Kaminer'schen Fälle ist sehr interessant und instructiv.

Zur Evidenz geht der Einfluss des Krankheitsgrades auf den Verlauf der Lungentuberculose nach der Entbindung namentlich hervor aus dem Material der künstlichen Schwangerschaftsunterbrechung, welches verhältnissmässig am meisten Angaben über das Stadium enthält. Wir zeigen dies in Folgendem an dem diesbezüglichen Material Kaminer's und an unseren Sammelberichten $D$ und $E$ des Literatur-Materials.

Der Ausgang obne Rücksicht auf den Krankheitsgrad bei Kaminer's Zusammenstellung lautet: Es waren arbeitsfäbig 10 $=67 \mathrm{pCt}$., nicht völlig arbeitsfähig $1=7 \mathrm{pCt}$., arbeitsunfähig $2=13$ pCt. der Frauen. Der Tod trat ein 2 mal, also in 13 pCt. der Fälle.

Mit Berücksichtigung des Stadiums ergeben sich folgende Resultate: Von 8 Frauen des ersten. Stadiums wurden arbeitsfähig $7=871 / 2 \mathrm{pCt}$., gestorben ist $1=12 \frac{1}{2} \mathrm{pCt}$. Es wurden von 7 Patientinnen des zweiten Stadiums arbeitsfähig $3=43$ pCt., nicht völlig arbeitsfähig $1=14$ pCt., nicht arbeitsfähig $2=29 \mathrm{pCt}$., gestorben ist $1=14 \mathrm{pCt}$. 
In einem Sammelbericht D stellten wir 32 Fälle von künstlichem Abort zusammen, in denen der Ausgang bekannt war.

Der Krankheitsgrad ist bei 10 Fraven nicht genannt. Es befanden sich von den übrigen Kranken 17 im ersten, 4 im zweiten, 1 im dritten Stadium.

Das endgültige Ergebniss aller 32 Fälle ohne Berücksichtigung des Stadiums war: Positiver Erfolg bei $27=84$ pCt., negativer bei $5=16$ pCt. Die 5 Fälle negativen Erfolges betreffen ebenso viele Todesfälle.

Von den verstorbenen Frauen befand sich je eine im ersten, zweiten und dritten Stadium, von den zwei anderen ist dasselbe nicht bekannt. Die positiven Erfolge betreffen demnach 16 Fälle des ersten und 3 des zweiten Stadiums. Mithin würden sich für die 22 Fälle, in welchen der Grad der Erkrankung bekannt ist, folgende Resultate ergeben: Von 17 Frauen des ersten Stadiums starb $1=6$ pCt., positiven Erfolg hatten $16=94$ pCt., von 4 des zweiten Stadiums starb $1=25$ CCt., positiv war das Resultat bei $3=75$ pCt. Die einzige Patientin des dritten Stadiums ist gestorben.

In 28 in unserem Sinne verwendbaren Fällen von künstlicher Frühgeburt des Sammelberichtes $E$ befand sich je eine der Kranken im ersten und zweiten Stadium, $6 \mathrm{mal}$ ist dasselbe unbekannt, die anderen 20 gehören dem dritten an.

Ohne Rücksicht auf den Krankheitsgrad ergeben sich folgende Daten über das Resultat: Der Erfolg war ein positiver in 7 pCt. oder 2 der Fälle, ein negativer bei $26=93 \mathrm{pCt}$. Letzterer betrifft ebenso viele Todesfälle.

Berücksichtigt man das Stadium, so lautet das Resultat: Bei je einer Frau des ersten und zweiten war der Erfolg ein positiver, bei den 20 Patientinnen des dritten ein negativer.

Endlich verweisen wir, um den Zusammenhang zwischen Krankheitsstadium und -Verlauf zu illustriren, auf die weiter unten folgenden Resultate des Materials der Züricher Universitäts-Frauenklinik, welches ungefähr in gleichem Verhältniss Kranke aus den verschiedensten Gesellschaftsklassen betrifft.

Die Bedeutung des Stadiums der Lungentuberculose in seinen Beziehungen zu den Generationsvorgängen ist wohl schon lange erkannt, wie weit man aber von einer allgemeinen und richtigen Würdigung derselben entfernt war und noch ist, geht unter Anderem klar hervor aus der Unsicherheit bei der Indicationsstellung zur 
künstlichen Unterbrechung der Schwangerschaft bei dieser Erkrankung, über die wir später sprechen werden. Es darf nicht unerwähnt bleiben, dass eine richtige Würdigung dieser Bedeutung auch für die Tuberculoseforschung im Allgemeinen allerdings eine Errungenschaft erst neueren Datums ist. Unseres Erachtens besteht aber kein Grund, die gewonnenen Erfahrungen nicht auch auf die Lungentuberculose gravider Frauen anzuwenden, zumal auch für die einzuschlagende Therapie.

Hatte die Ueberzeugung vom ungünstigen Einflusse der Generationsvorgänge auf die Lungentuberculose im Laufe der Zeit immer mehr und mehr sich Bahn gebrochen, so gelangte man gleichzeitig zur Erkenntniss, dass ausser diesen den übrigen bekannten ätiologischen Momenten der Lungentuberculose und den mit dem Geschlechtsleben des Weibes zusammenhängenden Störungen eine grosse Bedeutung in schlimmem Sinne zukomme.

Grosse Uebereinstimmung herrscht in Bezug auf den Einfluss gewisser Altersperioden; sie gipfelt in folgendem Satze: Die im dritten Decennium ihres Lebens stehenden Frauen sind durch die Generationsvorgänge ganz besonders gefährdet.

Von den 1035 Kranken des Literaturmateriales standen etwa $7 / 10$ im dritten Decennium, die anderen $3 / 10$ vertheilten sich auf das zweite, vierte und die erste Hälfte des fünften.

Es kann in Betracht kommen, dass die meisten Ehen in dieser Altersperiode geschlossen werden.

Auf die grosse Gefahr wiederholter und rasch aufeinanderfolgender Schwangerschaften und Geburten, besonders abnormer, ist von jeher allerseits hingewiesen worden.

Unzweifelhaft ist der Antheil an dem verderblichen Einfluss auf die Lungentuberculose der innerhalb der Generationsvorgänge auftretenden Complicationen.

Von denjenigen seitens der Schwangerschaft werden als am gefährlichsten diejenigen bezeichnet, welche auf Störungen der digestiven Functionen beruhen, vom einfachen, besonders länger anhaltenden Brechen bis zur ausgesprochenen Hyperemesis; von denjenigen seitens der Tuberculose namentlich die tuberculösen Kehlkopf- und Darmerkrankungen.

Wohl mit am unheilvollsten beeinflussen ungünstige Lebensverhältnisse mit ihren Consequenzen den Verlauf 
der Krankheit bei schwangeren und entbundenen Frauen, eine Thatsache, welche schon den älteren Aerzten (Capuron, Caresme, Coillot, Lassegue u. A.) bekannt war.

Unentschieden bleibt die Frage, ob der hereditären Belastung der ihr zugetheilte verderbliche Einfluss im Verlaufe der Generationsvorgänge zukommt oder nicht.

Es fehlt an Erfahrungon über den Verlauf der spontanen und künstlichen Entbindungen bei Phthisis florida. Wenn man die Machtlosigkeit jeglicher Therapie der wirklichen floriden Lungentuberculose gegenüber in Betracht zieht, so ist die Annahme wohl berechtigt, dass in solchen Fällen der Krankheitsprocess durch eine Intervention sich nicht wird aufhalten lassen.

Auf die Ergebnisse des von uns benutzten Literaturmaterials und der unten folgenden Fälle der Züricher Frauenklinik basirend, stellen wir hinsichtlich des Einflusses der Geburt, des Wochenbettes und der Folgezeit auf die Lungentuberculose, soweit ein Zusammenhang zwischen beiden besteht, folgenden Erfahrungssatz auf:

Der Verlauf und Ausgang der Lungentubercalose im Anschluss an den Geburtsact ist sowohl nach spontaner als künstlicher Entbindung, insofern die übrigen ätiologischen Momentesowohl von Seiten der Tuborculose als von Seiten des Geschlechtslebens des Weibes in Betracht gezogen werden, abhängig vom Stadium und Charakter der Erkrankungim Sinne der Erfahrungssätze, welche die Tuberculoseforschung für diese im Allgemeinen aufgestellt hat.

Daraus resultirt naturgemäss das Postulat: Bei der Betrachtung der Resultate sind die einzelnen Krankheitsstadien auseinanderzubalten.

Wir kommen auf die Rückwirkungen der Lungentuberculose auf die Generationsvorgänge zu sprechen und ergeben sich aus dem Gesammimaterial auch hier eine Reihe von Erfahrungssätzen, welche vorläufig als feststehend gelten dürfen:

Conceptionsfähigkeit besteht bei den Frauen jeden Stadiums.

Der Lungentuberculose kommt ein Einfluss auf die Gravidität insofern $z u$, als sie zur spontanen Unterbrechung derselben führen kann and zwar geschieht dies umso häufiger, je schwerer die Erkrankung ist und umgekehrt. Spontaner Abort tritt selten ein, häufiger die spontane Frühgeburt, zumal bei schwerer Tuberculose. 
Der Verlauf der Geburt wird durch die Lungentuberculose wenig beeinflusst.

Die Geburt erfolgt, abgesehen von anderen diese ungünstig beeinflussenden Factoren (Beckenenge etc.), meist rasch und leicht nach dem übereinstimmenden Urtheil der Beobachter, selbst in schweren Fällen. Dyspnoische und Schwächezustände, die das Mitpressen erschweren oder es unmöglich machen, Zustände, die namentlich bei schwerer Erkrankung in Betracht kommen, können sie selbstverständlich verlängern, Auftreten von Hämoptoe wurde von einzelnen Autoren relativ häufig beobachtet.

Die Kleinheit der Kinder und Schlaffheit der mütterlichen Weichtheile soll die Leichtigkeit der Entbindungen erklären.

Die Nachkommenschaft tuberculöser Frauen ist direct und indirect gefährdet.

Es wird bestätigt, dass diese Kinder, wenn sie, was allerdings selten vorkomme, nicht bereits vor der Geburt absterben, oft todt zur Welt kommen; dass diejenigen, welehe lebend das Licht der Welt erblicken, meist schwächlich, schlecht genährt und elend sind und ein grosser Theil derselben frühzeitig zu Grunde geht oder, wenn sie Jebensfähig bleiben, sie in einer späteren Lebensperiode der Drüsen- oder Lungentuberculose oder anderen intercurrirenden Krankheiten, zu welchen sie ganz besonders disponirt erscheinen, zum Opfer fallen, und zwar soll dies Schicksal auch solche treffen, welche kräftig und gut entwickelt sind und gesund scheinen. Dies soll die Regel sein.

Durch zuverlässige Beobachtungen ist ausser Frage gestellt, dass tuberculöse Mütter Kinder gebären, welche lebensfähig bleiben, nicht an Tuberculose erkranken und nützliche Mitglieder der menschlichen Gesellschaft werden.

Ja selbst schwerkranke, bis zum Skelett abgemagerte Frauen, sollen relativ häufig kräftige, gut entwickelte, selbst abnorm grosse und schwere Kinder gebären, allerdings im Verhältniss zur Gesammtzahl der Geburten in versehwindender Minderzahl.

Hecker und Buhl erklären dieses Vorkommniss für möglich dadurch, dass die fortdauernde Entziehung von Ernährungsmaterial, welche das Wachsthum des Kindes bedingt, bei Tuberculose eine sehr energische zu sein pflege.

Eine hervorragende Rolle wird in Bezug auf die Nachkommenschaft tuberculöser Mütter den socialen Verhältnissen zugeschrieben. Durch sofortige Isolirung, Pflege, Kuren soll es gelingen, einen 
grossen Theil von Kindern auch von in ärmlichen Verhältnissen lebenden Frauen zu erhalten.

Robert will bei den Früchten lungentuberculöser Frauen unvollkommen entwickelte Lungen gefunden haben.

Die Aufgabe, dem Schicksal der Kinder tuberculöser Mütter einschliesslich der Frühgeburtskinder nach spontanen und künstlichen Entbindungen nachzuforschen, wäre eine sehr wichtige und dankbare.

Um über den Einfluss der Iungentuberculose auf das Product der Conception zu sicheren Schlüssen zu gelangen, ist es erforderlich, Nachforschungen über das Schicksal der Kinder von Frauen aller Krankheitsgrade und Gesellschaftsklassen, auch der leichtkranken insbesondere anzustellen. Die wenigen in diesem Sinne verwendbaren Angaben ergeben auch hier die Abhängigkeit dieses Einflusses vom Grade der Erkrankung der Mutter, indem die Kinder schwerkranker Frauen mehr gefährdet erscheinen als diejenigen der leichtkranken.

Soweit hierüber Berichte vorlagen, wird von den Autoren auf die Unzulänglichkeit der Milch tuberculöser Wöchnerinnen, sowie auf die Möglichkeit der Infection des Kindes durch die Muttermilch verwiesen.

Die Untersuchungen über die congenitale Uebertragung der Tuberculose sind von einem Abschlusse weit entfernt. Wir haben diese Frage in unserer Arbeit nicht aufgenommen.

Der Symptomencomplex ist bei der Lungentuberculose schwangerer und entbundener Patientinnen derselbe, die Diagnose bietet dieselbe Schwierigkeit oder Leichtigkeit wie bei dieser Krankheit ausserhalb der Generationsvorgänge.

Es besteht kein Grund, die Erfahrungssätze über die Prognose bei Lungentuberculose im Allgemeinen auf den Verlauf derselben innerhalb der Generationsvorgänge nicht anzuwenden. Diese Prognose ist aber, alle anderen in Frage kommenden Factoren mitberücksichtigt, in hohem Grade abhängig vom Stadium und Charakter der Krankheit.

Die Erkenntniss der bisher geschilderten Wechselbeziehungen zwischen den Generationsvorgängen und der Lungentuberculose und vice versa musste nothwendigerweise die Aerzte veranlassen in therapeutischer Beziehung Stellung zu nehmen, keine leichte Auf- 
gabe, da sie nicht vom rein ärztlichen Standpunkte aus handeln durften, sondern juristische, theologische und Gesichtspunkte anderer Natur zu berücksichtigen hatten.

Das Bestreben, den unheilvollen Wirkungen durch möglichst wirksame prophylaktische Maassnahmen entgegenzutreten, führte zum Postulat des absoluten Eheverbotes nicht nur bei manifester, activer, sondern auch bei latenter und stationärer Lungentubereulose. Selbst prädisponirte weibliche Personen wurden von den Freuden des Mutterglückes und des Familienlebens ausgeschlossen.

Aehnliche Intentionen führten, wie Brehmer erzählt, im Jahre 1789 in Neapel und später in Portugal zur Erlassung eines Decretes zur Isolirung der Phthisiker. Eine Verminderung der Tubereulose fand jedoch nicht statt, weil die Verordnungen auf jegliche Weise umgangen wurden.

Weniger ausschliessliche Anhänger der Eheverweigerung wollen von einem absoluten Verbote, das doch nicht befolgt werde, inhuman und undurchführbar sei, absehen, knüpfen aber an die Ertheilung der Eheerlaubnis gewisse Bedingungen, unter andern folgende: Die Lungentuberculose muss vor derVerheirathungstationär oder ausgeheilt sein und zwar muss der stationäre Zustand oder die Heilung mindestens 1-2 Jahre andauernd bestanden haben, bevor die Ehe zu gestatten ist.

Weniger rigoros dürfe das Eheverbot auch gehandhabt werden nach dem Ueberschreiten des 30. Lebensjahres, bei Candidatinnen in guten Lebensverhältnissen und solchen mit leichter Erkrankung. Patientinnen mit vorgeschrittener, wenn auch stationärer Lungentuberculose, sei-der Consens zu verweigern. Kikuth will denselben nur den Patientinnen des ersten, höchstens des zweiten Stadiums ertheilen.

Der Curiosität wegen erwähnen wir im Gegensatz zu obigen Rathschlägen die Ansicht von Sims (z. n. Baumes), welcher die Ehe bei Phthise als ein meist sicher wirkendes "specifisches Mittel ${ }^{\text {" }}$ empfahl.

Kikuth äussert sich noch über einen Vorschlag die Ehe Tuberculöser betreffend, indem er sagt: „Neu ist der Gedanke die Ehe Tuberculöser zu empfehlen, durch welche mit der Zeit bei den kommenden Geschlechtern allmählich eine Immunität erzeugt werde. $"$

Statt practisch undurchführbarer Bestimmungen wird Aufklärung und Belehrung der Ehestandscandidaten und deren Familien fast 
384 Pradella, Künstliche Unterbrechung der Schwangerschaft.

allgemein verlangt, besonders Hinweis auf die Gefahren für den Patienten selbst und dessen Nachkommenschaft. Im Uebrigen solle, nachdem eine möglichst klare, sachliche Belehrung stattgefunden habe, die freie Entscheidung den Betheiligten überlassen werden, die persönlich dann die Verantwortlichkeit für ihr Handeln zu tragen haben.

Als vorbeugende Maassnahme, insbesondere bei Frauen, bei welchen erfahrungsgemäss die Generationsvorgänge einen verderblichen Einfluss auf die Lungentuberculose zur Folge gehabt hatten, wurde analog dem Ehe- das. Conceptionsverbot empfohlen. Abgesehen von der Nichtbefolgung der diesbezüglichen ärztlichen Vorschriften: Empfeblung der Abstinenz, des "Coitus interruptus", Verbot des "Congressus", Anwendung anticonceptioneller Mittel, wird als grosser Uebelstand empfunden, dass es an wirklich unschädlichen Mitteln zu letaterem Zwecke fehlt.

Auch hier wird offene gründliche Aufklärung der Betheiligten mit Hinweis auf die ernsten Folgen im Falle der Nichtbeachtung der ärztlichen Rathschläge anempfohlen.

Die Schwangerschaftserlaubniss soll erst dann ertheilt werden, wenn 1-2 Jahre lang dauernder Stillstand und Heilung des Lungenleidens vorhanden war.

Allseitiger Zustimmung erfreut sich in prophylaktischem Sinne die Nothwendigkeit des Stillverbotes bei tuberculösen Wöchnerinnen.

Wie aber solite der Arzt sich einer an Lungentuberculose leidenden Graviden gegenüber verhalten, passiv, abwartend oder activ, intervenirend? Darüber gehen auch heute noch die Meinungen weit auseinander und theilen die Aerzte sich in zwei grosse Partheien, in Gegner und Anhänger der künstlichen Unterbrechung, von welcher wir in der Folge auch kurzweg als Intervention, Operation, Eingriff sprechen werden.

Die Gegner einer künstlichen Unterbrechung berufen sich zunächst auf die Thatsache, dass erfahrungsgemäss an Lungentuberculose erkrankte schwangere Frauen, besonders bei sorgfältiger Ueberwachung, rechtzeitiger Beseitigung schädigender Momente und Einleitung einer rationellen Behandlung ohne Schaden für ihre Gesundheit die zeitige Geburt abwarten und überstehen können, zumal dann, wenn die Kranke auch nach der spontanen Entbindung der Vortheile einer guten Pflege und geeigneten Behandlung theilhaftig wird. Fin operativer Eingriff soll auf diese Weise vermieden werden können und wird die Behauptung aufgestellt, dass die Re- 
sultate bei solchem Verhalten nicht nur ebenso gute seien, sondern die der Intervention sogar übertreffen.

Manche liessen sich dabei auch von dem Gedanken leiten, dass, sollte das abwartende Verhalten nicht zum Ziele führen, dem Arzte das Mittel der Intervention immer noch zu Gebote stehe. So deuten wir Nagel's Ausspruch: "Die rationellste Therapie ist: die Krankheit zu behandeln und die Schwangerschaft zu schonen, und diese Therapie darf erst auf dringende Indication hin verlassen werden."

Die oft wenig erfreulichen Resultate des operativen Vorgehens in den allerdings meist schweren Fällen mochte den Vertretern des conservativen Standpunktes viele Anhänger zuführen.

Existe-t-il un fait, qui démontre d'une façon nette, que l'expulsion prématurée du produit de conception ait retardé l'evolution de la maladie et la terminaison fatale? Je n'en connais pas! En face d'ure femme tuberculeuse le médecin n'a qu'une chose à faire: soigner la tuberculeuse et surveiller simplement la grossesse. " So stellt und beantwortet Pinard die obige Frage, ohne freilich anzugeben, was der Arzt thun soll, wenn der Zustand der Kranken der Ueberwachung und Behandlung zum Trotz sich andauernd verschlimmert und das verhängnissvolle Ende unabwendbar erscheint, ausser er sei im ausschliesslichen Interesse des Kindes zu handeln entschlossen.

Si la mêre mourait sans être accouchée on aurait la ressource de l'operation césarienne ou l'accouchement forcé post mortem, lautet der wiederum nur das Wohl des Kindes berücksichtigende Vorschlag von Tarnier und Budin.

Von anderen Geburtshelfern, so von d'Outrepont und Stehberger wurde in hoffnungslosen Fällen die künstliche Unterbrechung der Gravidität zur Rettung des Kindes für indicirt erachtet. Die Stehberger'sche Indication bringt den erläuternden Zusatz am Schlusse: - als möglichsten Ersatz der Lex regia. Dem Sinne nach begegnen wir derselben Indicationsstellung bereits in der Inaugural-Dissertation des Gottlob Pfäfflin vom Jahre 1842, wo unter den Krankheiten schwangerer Fraven, welche die künstliche Frühgeburt indiciren, aufgezählt wird (Seite 21): „... endlich die Schwindsucht Schwangerer, von denen man glaubt, dass sie das Ende der Schwangerschaft nicht erreichen, um wenigstens das Kind zu retten, wozu nach den Zusammenstellungen von Riecke, Reinhardt u. a. durch den Kaiserschnitt an der Verstorbenen durchaus keine Wahrscheinlichkeit vorhanden ist." 
Die Gegner der Intervention führen als weitere Gründe gegen dieselbe ins Feld: die Gefahr des Eingriffs, die Unsicherheit des Verlaufes und der Prognose der Lungentuberculose, die Gefahr des Missbrauchs, die Möglichkeit des Conflictes mit dem Strafgesetzbuche, die Uneinigkeit bezüglich der Indicationsstellung und Indicationsbegründung seitens der Anhänger eines activen Vorgehens etc.

Die Freunde des operativen Eingriffs, von denen eine kleine Zahl, gleich wie ihre Gegner, in erster Linie und vor Einleitung der Operation eine rationelle Behandlung der Lungentuberculose verlangen, berufen sich auf die, wenn auch spärlichen, positiven Erfolge derselben und halten ihrerseits den Anhängern des conservativen Standpunktes die ungünstigen Resultate dieser Therapie entgegen. Die Unsicherheit der Prognose- und Mannigfaltigkeit des Verlaufes komme in gleicher Weise bei abwartendem Verhalten in Betracht. Der operative Eingriff als solcher biete heutzutage kaum mehr eine Gefahr. An die tuberculöse Grạida stelle die spontane zeitige Geburt viel höhere Anforderungen als die künstliche Entbindung, gegen eventuellen Missbrauch und dem Richter gegenüber vermöge der Arzt sich wirksam zu schützen ete.

Bei aller Uebereinstimmung, dass die künstliche Unterbrechung der Schwangerschaft der abwartenden Methode vorzuziehen sei, sind die Meinungen ihrer Anhänger über die Indicationsstellung sehr getheilte, zum Theil einander direct entgegenstehende. Wir beschränken uns darauf, die hauptsächlichsten Indicationen namhaft zu machep.

Sofort nach Stellung der Diagnose Tuberculose verlangen Maragliano und seine Anhänger Acconci, Cuzzi, Kikuth, Hamburger (bei Arbeiterfrauen) die Intervention. Nur als „ultimum refugium", wenn die Lungentuberculose trotz sorgfältiger Ueberwachung und rationeller Behandlung fortschreitet oder eine solche Behandlung undurchführbar ist, soll sie nach anderen gestattet sein.

Der symptomatischen Indication, die man zur Bekämpfung lästiger, quälender Beschwerden, wie Husten, Athemnoth, Schmerzen, Brechen will gelten lassen, wird die vitale Indication gegenübergestellt zur Rettung des Lebens der Mutter nur bei directer unzweifelhafter Lebensgefahr.

Im Gegensatz zur vitalen Indication soll die Intervention bei indirecter Bedrohung der Gesundheit und des Lebens der Mutter eintreten. 
Wollen die Einen nur in prognostisch günstigen Fällen, wo andauernde Besserung, Stillstand, Heilung zu erwarten sind, also vorwiegend bei beginnender oder leichter Erkrankung eingreifen, so halten andere ein Einschreiten nur bei allerschwerster, ja hoffnungsloser Erkrankung der Frauen für angezeigt.

Die Progressivität der Krankheit während der Schwangerschaft, deren sicher diagnosticirtes Vorhandensein ron manchen als unerlässliche Vorbedingung angesehen wird, braucht nach der Ansicht anderer Autoren nieht vorhanden za sein. Diese. letzteren wollen die Gravidität auch bei stationärer Lungentuberculose unterbrechen, namentlich wenn vorhergegangene Schwangersehaften, Geburten oder Wochenbetten einen ungünstigen Einfluss auf die Erkrankung ausgeübt hatten.

Complication der Lungentuberculose, insbesondere mit Kehlkopf-, Darmtuberculose oder Hyperemesis gravidarum, gilt einerseits als absolute Indication, während andere in solchen Fällen nie operiren würden.

Der Indicationsstellung im Interesse der Mutter steht diejenige im Interesse des Kindes und zwar oft schroff gegenüber, während vermittelnde Vorschläge die beiderseitigen Interessen berücksichtigt wissen wollen.

Fügen wir noch bei, dass von Veit und Ascoli die Entscheidung der Frage der Intervention vom Verhalten des Körpergewichtes während der Gravidität abhängig gemacht wird, dass bei Frauen, bei welchen die tuberculöse Erkrankung kurz vor oder während der Schwangerschaft erstmals auftrat, der Eingriff ganz besonders indicirt sei, dass manche bei Multiparen eher als bei Primiparen zur Operation rathen, dass endlich bei Geschiedenen und Ledigen davor gewarnt wird, so glauben wir die wichtigsten Indicationen genannt zu haben.

Auf die Begründung derselben hier näher einzugehen würde zu weit führen.

Die principielle Empfehlung der künstlichen Unterbrechung der Schwangersehaft bei Lungentuberculose wird von der grossen Mehrheit der Aorzte abgelehnt und die Entscheidung von Fall zu Fall empfohlen.

Ausserordentliche Schwierigkeiten bereitete die Frage nach dem günstigsten Zeitpunkte der Operation. Je nach der Lage des Einzelfalles wurde von den meisten Autoren bald die künstliche Fehl-, bald die artificielle Frühgeburt für indicirt erachtet, während 
manche nur die Ausführung der ersteren, andere nur die Anwendung letzterer empfahlen.

Die Anhänger des künstlichen Abortes konnten nur das Interesse der Mutter im Auge haben, deren Gesundheit und Leben in erster Linie zu berücksichtigen und höher zu werthen sei, als das kindliche, das erst in zweiter Linie in Betracht komme, da, wenn auch nicht alle Kinder, so doch die Mehrzahl elend und schwächlich zur Welt komme und, obwohl zum Theil gut und kräftig entwickelt, ein grosser Procentsatz früher oder später erkranke und zu Grunde gehe oder wenigstens den Keim der Krankheit auf die Nachwelt übertrage.

Mit der Prognose der Mutter verschlechtere sich auch diejenige des Kindes, könne der Arzt nicht beide erhalten, so solle er in erster Linie die Matter retten.

Dem künstlichen Abort wird ferner der Vorzug gegeben, weil der schädliche Einfluss auf die Krankheit in der ersten Hälfte der Gravidität stärker sein soll, als in der zweiten und zwar könne derselbe umso weniger sich geltend machen, je kürzer die Schwangerschaft dauere.

Besonders hervorgehoben wird, dass der künstliche Abort heutzutage kaum mehr eine Gefahr biete und ein leichterer Eingriff sei als die künstliche Frühgeburt, welche mehr der normalen Geburt gleichkomme und fast dieselben Ansprüche und Anforderungen an die Frauen stelle, wie diese.

Eine möglichst frühzeitige Intervention wird besonders bei in ärmlichen Verhältnissen lebenden Patientinnen für nothwendig erachtet. Wollen die einen nur interveniren, wenn durch den Nachweis ron Tuberkelbacillen die Diagnose der Lungentuberculose gesichert ist, so sind andere zum Eingriffe bereit, bevor noch Bacillen im Auswurf zu finden sind, sobald die Diagnose auf andere Weise zweifellos festgestellt ist.

Kikuth stellt zur Ausführung der Operation an die Patientin die Bedingung, dass sie sich zu einer hygienisch-diätetischen Cur nach der Entbindung verpflichte zur Ausheilung der Tuberculose.

Der künstliche Abort wird vielfach angefochten. Mit zahlreichen anderen Autoren verwirft Fritsch ihn absolut bei nicht complicirter Phthise, indem er sagt: "Ich erkläre mich also nie bereit zum Abort auf die Indication: Lungentubereulose".

Die Erhaltung and Rettung von Mutter und Kind soll nach der Meinung ihrer Anhänger in erster Linie der Zweck der künst- 
lichen Frühgeburt auch bei Lungentuberculose sein, jene Fälle ausgenommen, wo bei absolut hoffnungsloser Erkrankung der Mutter die Indication im alleinigen Interesse des Kindes zu stellen sei.

Es besteht aber, wie wir feststellen müssen, fast allgemein das Bestreben, den Zeitpunkt im Interesse des Kindes soweit wie möglich hinauszuschieben, selbst bis zu einem Termin, welcher auch nur die Möglichkeit eines lebend zu erhaltenden Kindes bietet, ohne dass in gleichem Maasse die Prognose der Mutter berücksichtigt wird, so dass die Operation thatsächlich nicht im Interesse beider, sondern im vorwiegenden Interesse des Kindes ausgeführt wird.

Das Bestreben, den Zeitpunkt der Operation in manchen Fällen vielleicht unwillkürlich zu Gunsten des Kindes zu verschieben, wird wohl durch die Thatsache erklärlich, dass doch manche tuberculöse Frauen gesunde und gesund bleibende Kinder gebären, welche nützliche Mitglieder der menschlichen Gesellschaft werden.

Andererseits scheint der Glaube an die Unheilbarkeit der Phthise im Sinne Virchow's noch weit verbreitet $z u$ sein und auch die Indicationsstellung bei dieser Krankheit naturgemäss $\mathrm{zu}$ beeinflussen, wenn auch nicht alle Autoren so weit gehen wie Tarnier und Budin, welche der Meinung sind, dass nur das kindliche Leben einen gewissen socialen Werth besitze, da die Mutter doch unheilbar verloren sei, oder wie Jaffé, welcher den Anhängern des künstlichen Abortes entgegenhält, es erscheine ihm nicht berechtigt, ein kindliches Leben zu opfern, um ein sicher verlorenes mütterliches nicht etwa ganz zu retten, sondern nur zu verlängern.

Suchen wir nach den Gründen der Uneinigkeit in Bezug auf die Indicationsstellung, so scheinen sie uns - wir haben einige derselben schon angedeutet - verschieden zu sein. Der innere Mediciner ist geneigt in dem Bestreben, die verderbliche Krankheit mit möglichst wirksamen Mitteln zu bekämpfen, die Indicationsstellung in diesem Sinne zu beeinflussen, ein Bestreben, das leicht zur Unterschätzung des kindlichen Lebens führt. Andererseits zieht der Geburtshelfer in seinem Bemühen, wenn immer möglich, ein lebendes Kind zu erhalten, die über die Lungentuberculose im Allgemeinen gewonnenen Erfahrungen und gewisse Eigenthümlichkeiten, welche dieser Krankheit als Complication der Schwangersehaft zukommen, bei der Stellung der Indication nicht 
genügend mit in Rechnung, was eine Unterschätzung der Interessen der Mutter zur Folge haben kann.

"Der Geburtshelfer muss in erster Linie sein „inneres Arztthum" zu Rathe ziehen und erst, nachdem dieses gesprochen und den Befehl zum Handeln ertheilt hat, sein "Geburtshelferthum" zur That schreiten lassen", schreibt W. A. Freund ebenso kurz als treffend.

Eine erspriessliche Lösung der so wichtigen Streitfrage wird, um mit Freund zu sprechen, nur durch ein Zusammengehen und -wirken des inneren Arztthums mit dem Geburtshelferthum zu Stande kommen.

Der tiefere Grund, warum die Frage einer befriedigenden Lösung so lange harrt, beruht wohl in der Schwierigkeit und Unsicherheit der Prognosestellung bei Lungentuberculose. Wir werden auf diesen Punkt weiter unten zurückkommen.

Der Eingriff selber der künstlichen Unterbrechung der Gravidität wurde nach den auch sonst bekannten und üblichen Methoden ausgeführt. Wegen der Verlängerung der Wehenthätigkeit und zur Vermeidung von Krampfwehen wird vor künstlichem Blasensprung ausdrücklich gewarnt.

Sehr ängstlich sind viele Aerzte bezüglich der Narkose, die sich bei Lungentuberculose gewöhnlich von selbst verbieten solle.

Der Streit um die Berechtigung oder Nichtberechtigung der Intervention vom medicinischen Standtpunkte aus basirt auf denselben Gründen, auf welche die Uneinigkeit der Indicationsstellung zurückzuführen ist, die wir bereits angeführt haben. Auch die Berechtigung zur Ausführung der künstlichen Sterilisation wird beanstandet.

Uns beschäftigt noch kurz die juristische Seite der Frage, soweit diese die künstliche Fehlgeburt betrifft. So alt wie die Frage nach der Berechtigung des künstlichen Abortes zu therapeutischen Zwecken, so alt sind die Bedenken, die Unsicherheit, die Mahnungen zur Vorsicht dem Strafgesetzbuch und Richter gegenüber. Es wird ärztlicherseits nach bestimmten Indicationen gerufen, auf welche man sich berufen könne. Wohl wird darauf hingewiesen, dass die pflichtgetreve Ausübung des ärztlichen Berufes, welche die Erhaltung der Gesundheit und des Lebens der Mutter zum Zwecke hat, nicht unter den Begriff einer rechtswidrigen Handlung fallen könne; dass ferner zum Begriffe des Verbrechens der Dolus und die Heimlichkeit gehöre und dass endlich die Absicht, eine verbrecherische 
Handlung begehen zu wollen, erst zu beweisen sei. Trotzdem besteht ein Gefühl der Unsicherheit vor den gesetzgeberischen Bestimmungen, welches die Aerzte schon lange veranlasste, zu ihrem Schutze gewisse Maassnahmen zu treffen.

Man machte die Ausführung der Operation von gewissen Vorbedingungen abhängig. Solche waren: Beiziebung eines oder namentlich bei künstlichem Abort - mehrerer sachkundiger Aerzte behufs Consilium, Begutachtung durch autoritative Gutachten, schriftliche Protokollaufnahme, Einwilligung der Betheiligten und deren Angehörigen. Ja sogar die Beiziehung des Physicus als officieller gerichtlicher Sachverständiger wurde vorgeschlagen.

Von den Gegnern des künstlichen Abortes wird namentlich auch die Gefahr des Missbrauches als Argument dagegen aufgestellt, dem gegenüber die Anhänger betonen, dass der Arzt zur Vornahme der Operation nie gezwangen werden kann und dass er, wenn eine in Frage kommende Frau unter Nichtachtung der ärztlichen Rathschläge, nach vorhergegangener künstlicher oder spontaner Entbindung, wieder schwanger wurde, die Operation je nach Gutdünken ausführen oder verweigern kann.

Kaminer stellt für den künstlichen Abort folgenden Satz auf: „Der Arzt ist häufig berechtigt, nie verpflichtet, den künstlichen Abort bei Lungentuberculose einzuleiten".

Selbstverständlich war auch die Stellung der Kirche und zwar der katholischen - die protestantische scheint sich zur Frage nicht ausgesprochen zu haben - der Streitfrage gegenüber von grosser Tragweite aus Gründen, die wir unerörtert lassen. Wir begnügen uns festzustellen, dass der päpstliche Stuhl sich gegen die Zulässigkeit der künstlichen Unterbrechung der Schwangerschaft vor erreichter Lebensfähigkeit des Kindes stets ausgesprochen hat, obwohl seitens einiger Moraltheologen das Argument gelten gemacht wurde, das auch von juristischer Seite herangezogen wird, dass die Mutter sich im Zustande der Nothwehr befinde.

Damit schliessen wir den Rückblick auf die Literatur und lassen in kurzem Auszuge die Fälle der Züricher UniversitätsFrauenklinik folgen:

1. Fall. Hausfrau, 23 jährig. V para, hereditär belastet. Verschlimmerung der tuberculösen Lungenaffection I. Stadiums durch die bestehende Schwangerschaft. Einleitung des künstlichen Abortes im III. Monat. Besserung im Wochenbett. Weiterer Verlauf: Andauernde Besserung, Stillstand der Lungentuberculose, Arbeitsfähigkeit, also positiver Erfolg laut Bericht des Gatten vom 23. 12. 1905: „Nach jener vor- 
zeitigen Geburt ist das Lungenleiden vollständig zum Stillstand gekommen. Ebenfalls ist meine Frau nach 6 Monaten arbeitsfähig gewesen für Haus-, Garten- und Feldarbeit." Ausgang laut obigem Bẹricht: Die Frau hat in der Folge noch 4 zeitige Geburten und 1 spontanen Abort durchgemacht. und zwar in den Jahren 1894, 1896, 1897, 1899, 1900, ohne dass dieselben auf das Lungenleiden einen schädlichen Einfluss ausgeübt hätten. Patientin lebt und ist arbeitsfähig $12^{1 / 2}$ Jahre nach dem künstlichen Aborte.

2. Fall. Wäscherin, 35jährig. Vpara, hereditär belastet. Verschlimmerung der tuberculösen Lungenaffection I. Stadiums durch die bestehende Schwangerschaft. Finleitung des künstlichen Abortes im III. Monat. Besserung im Wochenbett. Weiterer Verlauf: Andauernde Besserung, Stillstand der Lungentuberculose, Arbeitsfahigkeit, also positiver Erfolg laut Anamnese und Untersuchung des Arztes, Dr. Pradella, vom 29. 7. 1900. Ausgang laut diesem Bericht: Der Erfolg war ein andauernder bis zur nächsten Gravidität im Jahre 1894, während welcher wieder Verschlimmerung des Lungenleidens eintrat. Beim Wiedereintritt in die Klinik befand Patientin sich noch im I. Stadium der Krankbeit. Ein am 30. 7. 1894 im LV. Monat eingeleiteter künstlicher Abort hatte wiederum positiven Erfolg, welcher bis zur nächstfolgenden Schwangerschaft im Jahre 1896 anhielt. Nach anfänglich eingetretener Verschlimmerung trat durch Schonung, Pflege und Kuren subjectiv Besserung ein. Die Geburt (Zwillinge, welche bald starben) verlief spontan. Die Frau erholte sich gut und war nachher wieder arbeitsfähig. Die objective Untersuchung der Lungen vom 29. 7. 1900 ergab stationäre Lungentuberculose, aber II. Stadiums. Laut Bericht der Heimatbehörde vom 16. 12. 1905 lebt Patientiu und ist als Wasscherin and Pensionshalterin arbeits- und erwerbsfähig - 12 Jahre 3 Monate nach dem ersten künstlichen Aborte.

3. Fall. Seidenzettlerin, 29jährig. II para, hereditär nicht helastet. Verschlimmerung der tuberculösen Lungenaffection II. Stadiums durch. die bestehende Schwangerschaft. Complication: Erbrechen. Einleitung. des künstlichen Abortes im IV. Monat. Besserung im Wochenbett. Weiterer Verlauf: Andauernde Besserung, Stillstand der Lungentuberculose, Arbeitsfähigkeit, also positiver Erfolg laut Anamnese und Untersuchung des Arztes, Dr. Pradella, vom 4. 2. 1906. Ausgang laut diesem Bericht: Patientin abortirte 1899 und 1902 ohne schädliche Folgen für ihr Lungenleiden. Die Untersuchung vom 4. 2. 1906 ergab stationäre Lungentubereulose II. Stadiums und ist die Frau voll arbeitsfähig - 10 Jahre 7 Monate nach dem künstlichen Aborte.

4. Fall. Hausfrau, 44jährig. XI para, hereditär belastet. Verschlinmerung der tuberculösen Lungenaffection I. Stadiums durch die bestehende Schwangerschaft. Einleitung des künstlichen Abortes im III. Monat. Besserung im Wochenbett. Weiterer Verlauf: Andauernde Besserung, Stillstand der Lungentuberculose, Arbeitsfähigkeit, also positiver Erfolg laut Anamnese und Untersuchung des Arztes, Dr. Pradella, vom 29. 7. 1900. Ausgang laut diesem Bericht: Der Erfolg dauerte an bis zur nächstfolgenden Schwangerschaft im Jahre 1896, in welcher wieder Verschlimmerung eintrat, so dass die Lungentuberculose in das II. Stadium überging. Ein am 1. 5. 1896 im III. Monat eingeleiteter künstlicher Abort erzielte positiven Erfolg, welcher bis zur Untersuchung vom 29. 7. 1900 andauerte. Diese ergab stationäre Lungentuberculose II. Stadiums bei voller Arbeitsfähigkeit. Patientin 
lebt bei abnehmender Arbeitskraft (541/2 Jahre alt); die Lungentuberculose ist stationär geblieben, beziehungsweise ausgebeilt - 101/2 Jahre nach dem ersten künstlichen Aborte laut Bericht der Kranken vom 8. 12. 1905: "Im Winter 1904 musste ich wegen heftigem Husten Herrn Dr. S. zu Rathe ziehen. Derselbe sagte mir, er hätte niemals geglaubt, dass meine Lungen so schön ausheilen würden. Arbeitskraft hat viel nachgelassen." Die Frau hatte nicht mebr geboren.

5. Fall. Hausfrau, 41jährig. IV para, hereditär belastet. Verschlimmerung der tuberculösen Lungenaffection II. Stadiums durch die bestehende Schwangerschaft. Einleitung des künstlichen Abortes im IV. Monat. Besserung im Wochenbett. Weiterer Verlauf: Andauernde Besserung, Stillstand der Lungentuberculose, Arbeitsfähigkeit, also positiver Erfolg laut Bericht des Arztes, Dr. B. Giesker, vom 28. 12. 1905: "Ich habe seiner Zeit den Eindruck gehabt, dass die künstliche Frühgeburt hier lebensrettend gewirkt hat, wenigstens machte der tuberculöse Prozess rasch Halt, während er in den ersten Monaten der Schwangersçhaft wahrscheinlich stark sich ausgedehnt hatte." Ausgang lant diesem Berichte: Patientin blieb arbeitsfähig bis zum November 1905 und lebt noch - 9 Jahre 9 Monate nach dem künstlichen Aborte. Im November 1905 erkrankte sie an einem Recidiv der Lungentuberculose nach Influenza. Die Frau hat nach der künstlichen Entbindung nicht mehr geboren.

6. Fall. Hausfran, 27jährig. III para, hereditär nicht belastet. Verschlimmerung der tuberculösen Lungenaffection II. Stadiums durch die bestehende Schwangerschaft, welche anfänglich mit Brechen complicirt war. Einleitung des künstlichen Abortes im III. Monat. Besserung im Wochenbett. Weiterer Verlauf: Vorübergehende Besserung, andauernde Verschlimmerung, Fortschreiten der Lungentubereulose, Arbeitsunfähigkeit, Tod, also negativer Erfolg laut mündlichem Bericht des Gatten vom Juli 1900 an Dr. Pradella. Ausgang laut diesem Berichte: Der Tod erfolgte am 31. 8. 1899 - 2 Jahre 9 Monate nach der künstlichen Entbindung.

7. Fall. Hausfrau. 30jährig. IV para, hereditär nicht belastet. Verschlimmerung der tuberculösen Lungenaffection II. Stadiums durch die bestehende Schwangersehaft. Am 12. 12. 1896 war die Patientin klinisch vorgestellt worden, empfohlen zur Einleitung des künstlichen Abortes. Es wurde beschlossen, zuzuwarten, um ein lebensfähiges Kind zu bekommen. Beim Wiedereintritt in die Klinik wurde Uebertritt der Erkrankung vom damals bestehenden I. ins II. Stadium festgestellt. Einleitung des künstlichen Abortes im VII. Monat am 28. 1. 1897, also 11/2 Monate nach der klinischen Vorstellung. Erst Verschlimmerung, dann Besserung im Wochenbett. Weiterer Verlauf: Andauernde Besserung, Stillstand der Lungentuberculose, also positiver Erfolg laut Bericht des Arztes, Dr. Werner, vom 28. 12. 1905: „ad 1: bis August (ist das Lungenleiden) gleich geblieben". und des Gatten vom 19. 12. 1905: „Nach der Geburt war es dann besser und konnte sie im Frühjahr und Sommer wieder spazieren gehen, aber Ende Sommer wurde sie schwächer und musste endlich immer im Bett bleiben." Ausgang laut obigem Bericht des Arztes: „Dann (August) Verschlimmerung bis zum exitus am 5. 9. 1897." Also tödtlicher Ausgang - 7 Monate nach der künstlichen Entbindung.

8. Fall. Hausfrau, 29jährig. IIIpara, hereditär belastet. Verschlimmerung der tuberculösen Lungenaffection III. Stadiums durch die 
bestehende Schwangerschaft. Einleitung des künstlichen Abortes im II. Monat. Besserung im Wochenbett. Weiterer Verlauf: Besserung vorübergehend, andauernde Verschlimmerung, Fortschreiten der Lungentuberculose, Arbeitsunfähigkeit, Tod, also negativer Erfolg laut mündlichem Bericht des Gatten an Dr. Pradella vom 29. 7. 1900: Nach dem Austritt aus der geburtshilflichen Klinik entwickelte sich eine Mastdarmfistel, nach deren Operation der Verlauf bis zum Tode ein rapider war. Vier Wochen nach der Entlassung aus der Klinik hatte Patientin sich einer Baunscheidt'schen Kur unterzogen. Ausgang laut obigem Bericht: Der Tod erfolgte - 6 Monate nach der künstlichen Entbindung.

9. Fall. Hausfrau, 23 jährig. II para, hereditär belastet. Verschlimmerung der tuberculösen Lungenaffection I. Stadiums durch die bestehende Schwangerschaft. Einleitung des künstlichen Abortes im IV. Honat. Besserung im Wochenbett. Weiterer Verlauf: Andanernde Besserung, Stillstand der Lungentuberculose, Arbeitsfähigkeit, àlso positiver Erfolg laut mündlichem Berichte der Patientin an den Arzt, Dr. Pradella, vom 30.7. 1900, welchem zu entnehmen war, dass bereits im Jahre' 1896 wegen Lungentuberculose eine künstliche Fehlgeburt mit positivem Erfolge eingeleitet worden war. Ausgang laut Bericht des Arates, Prof. Cloetta, vom 17. 11. 1905: Im Jahre 1900 wurde Patientin wieder gravid. Es wurde bei der Kranken, welche sich im II. Stadium der Lungentuberculose befand, der künstliche Abort eingeleitet. Nach vorübergehender kleiner Verschlechterung erbolte sich die Frau und war arbeitsfähig. 1902 trat wieder Schwangerschaft ein. Es wurde nochmals die künstliche Fehlgeburt eingeleitet: „diesmal aber mit viel besserem Resultat. Ich habe dann die Patientin 2 Jahre nicht mehr gesehen und erst vor einigen Tagen hat sie sich wieder einmal gestellt und konnte eine entschiedene Besserung constatirt werden und Neigung zur Narbenbildung. Während der ganzen Zeit hat die Fran immer gearbeitet." Patientin lebt demnach, ist arbeitsfähig und zeigt die Lungentuberculose, entschieden gebessert, eine Neigung zur Narbenbildung - 8 Jahre 1 Monat nach der künstlichen Entbindung in der Klinik (9 Jahre 1 Monat nach dem ersten künstlichen Abort).

10. Fall. Hausfrau, 30 jährig. I para, hereditär belastet. Verschlimmerung der tuberculösen Lungenaffection I. Stadiums durch die bestehende Schwangerschaft, welche noch durch beginnende Luingentuberculose und Coxitis tuberculosa dextra complicirt ist. Finleitung des künstlichen Abortes im III. Monat. Erst Verschlimmerung, dann Besserung im Wochenbett. Weiterer Verlauf: Andauernde Besserung, Stillstand der Lungentuberkulose, Arbeitsfähigkeit, also positiver Erfolg laut Bericht des Gatten vom 10. 8. 1900 und des Arztes, Dr. Keller, vom 15. 12. 1905: „Fragliche Patientin habe ich seit drei Jahren nicht mebr gesehen, vorher nur ab und zu .....'Jedesmal konnte ich constatiren, dass das Lungenleideu keine oder wenigstens nicht nachweisbare Fortschritte gemacht hatte." Patientin hat nicht wieder geboren. Ausgang laut Bericht der Heimathbehörde vom 14. 12. 1905: Patientin lebt und ist erwerbsfähig - $6^{3} / 4$ Jahre nach der künstlichen Entbindung.

11. Fall. Arztsgattin, 21 jährig. IIpara, hereditär belastet. Verschlimmerung der tuberculösen Lungenaffection I. Stadiums durch die hestehende Schwangerschaft. Complication: starke Anämie. Einleitung des künstlichen Abortes im III. Monat. Besserung im Wochenbett. 
Weiterer Verlauf: Andauernde Besserung, Stillstand der Lungentuberculose, gute Leistungsfähigkeit, also positiver Erfolg laut Bericht des Arztes, Hofrath Turban, vom 22. 11. 1905. Ausgang laut diesem Bericht: "Nach der Entlassung keine direkten Nachrichten. Patientin, eine leichtsinnige, unvorsichtige Person, soll bald nach der Heimkehr ein Recidiv bekommen und gestorben sein. Da die Patientin am 27. 11. 1899 Turban's Sanatorium verliess, so war der positive Erfolg mindestens 6 Monate nach dem künstlichen Aborte noch vorhanden.

12. Fall. Hausfrau, 32jährig. III para, hereditär belastet. Verschlimmerung der tuberculösen Lungenaffection I. Stadiums (beim Eintritt in die Klinik) durch die bestehende Schwangerschaft. Einleitung des künstlichen Abortes im V. Monat. Besserung im Wochenbett. Weiterer Verlauf: Andauernde Besserung, Stillstand der Lungentuberculose, Arbeitsfähigkeit, also positiver Erfolg laut Bericht des Arztes, Dr. Schönemann, vom 31. 7. 1900: „Die damalige hochgradige (?) tuberculöse Affection ist also auf ein Minimum zurückgegangen. Subjectives Befinden deshalb auch gut. Abendtemperaturen und Schweisse gleich Null." Ausgang laut Bericht des Gatten vom 14. 12. 1905: Dieser Bericht bestätigt zunächst den positiven Erfolg der Operation. Im August 1902 bekam die Patientin ein Recidiv der Krankheit, welchem sie rasch zum Opfer fiel - 3 Jahre 1 Monat nach der künstlichen Entbindung.

13. Fall. Kaufmannsgattin, 23jährig. II para, hereditär nicht belasstet. Verschlimmerung der tuberculösen Lungenaffection III. Stadiums durch die bestehende Schwangerschaft. Complication: Beginnende Kehlkopftuberculose. Einleitung des künstlichen Abortes im V. Monat. Besserung im Wochenbett. Weiterer Verlauf: Andauernde Besserung. Stillstand der Lungentuberculose, Leistungsfähigkeit dem Stadium entsprechend gut laut Bericbtes des Arztes, Dr. Pradella, nach der Untersuchung rom 6.5. 1900. Ausgang laut diesem Berichte: Patientin unterbrach trotz dringenden Abrathens die Kur in Davos, reiste nach Hause, lebte dort wie eine Gesunde, bekam ein Recidiv und starb trotz aller Kuren am 16. 12. 1902 - 2 Jahre 11 Monate nach der künstlichen Entbindung.

14. Fall. Waschfrau, 33jährig. IIIpara, hereditär belastet. Verschlimmerung der tuberculösen Lungenaffection III. Stadiums durch die bestehende Schwangersehaft. Complicationen: Anfänglich Erbrechen, Pleuritis exsudativa. Einleitung des künstlichen Abortes im V. Monat. Besserung im Wochenbett. Weiterer Verlauf: Vorübergehende Besserung, Verschlimmerung, Fortschreiten der Lungentuberculose, Arbeitsunfähigkeit, Tod, also negativer Erfolg laut Bericht des Arztes, Dr. Suter, vom 19. 11. 1905: "Allein die Tuberculose der Lungen wurde dadurch (den künstlichen Abort) nicht aufgehalten, sie schritt unaufhaltsam weiter und nach etwa 1/2 Jahre erlag die Frau ihrem Leiden." Ausgang laut diesem Bericht und laut Anamnese und Untersuchung des Arztes, Dr. Pradella, vom 30. 7. 1900, welch' letztere den negativen Erfolg bestätigte. Patientin, in sehr ärmlichen Verhältnissen lebend, musste sofort nach der Entlassung aus der Klinik die Hausgeschäfte besorgen und als Wäscherin für ihre Familie das Brod verdienen.

15. Fall. Hausfrau, 31jährig. IV para, hereditär nicht belastet. Keine Verschlimmerung der tuberculösen Lungenaffection II. Stadiums durch die bestehende Schwangerschaft objectiv nachweisbar. Subjectives 
Befinden schlecht. Einleitung des künstlichen Abortes im IV. Monat. Besserung des Allgemeinbefindens und des Lungenzustandes im Wochenbett. Weiterer Verlauf: Andauernde Besserung, Stillstand der Lungentuberculose, Arbeitsfähigkeit, also positiver Erfolg laut Bericht des Arztes, Dr. Staub, vom 25. 11. 1905: „Kam in sehr gutem Zustande aus der Frauenklinik zurück, machte hier (im Sanatorium Wald) weitere Fortschritte; am 18. 12. als voll arbeitsfähig und ohne Tuberkelbacillen im Sputum entlassen." Ausgang laut obigem Bericht: "Leider entzog sich die Patientin unserer weiteren Beobachtung und konnte der Aufenthalt nicht mehr in Erfahrung gebracht werden." Der positive Erfolg war also mindestens 2 Monate nach dem künstlichen Aborte noch festzustellen.

16. Fall. Hausfrau, 32jährig. IV para, hereditär nicht belastet. Verschlimmerung der tuberculösen Lungenaffection III. Stadiums durch die bestehende Schwangerschaft. Complication: Kehlkopftuberculose. Einleitung der künstlichen Frühgeburt im X. Monat. Verschlimmerung im Wochenbett. Weiterer Verlauf: Andauernde Verschlimmerung, Fortschreiten der Lungentuberculose, Arbeitsunfähigkeit, Tod, also negativer Erfolg laut Bericht des Gatten vom 27. 12. 1905. Ausgang laut diesem Berichte: Der Tod erfolgte am 30. 1. $1902-5$ Wochen nach der künstlichen Entbindung.

17. Fall. Hausfrau, 24jährig. IV para, hereditär belastet. Verschlimmerung der tuberculösen Lungenaffection I. Stadiums durch die bestehende Schwangerschaft objectiv nicht nachweisbar. Patientin hatte blutigen Auswurf, das subjective Befinden war schlecht. Einleitung des künstlichen Abortes im V. Monat. Besserung des Allgemeinbefindens im Wochenbett. Weiterer Verlauf: Andauernde Besserung, andauernder Stillstand der Lungentuberculose, Arbeitsfähigkeit, also positiver Erfolg lant Bericht des Arztes, Dr. Staub, vom 15. 12. 1905: "Lungenbefund genau wie früher, eine Verschlimmerung ist objectiv nicht nachweisbar. Seither ist die Patientin stets leistungsfähig geblieben.". Ausgang laut Bericht der Patientin vom 13. 12. 1905: Patientin theilt mit, dass die Besserung eine derartige war: „dass ich mich nun meiner besten Gesundbeit erfreuen kann," - 3 Jahre 9 Monate nach der künstlichen Entbindung.

18. Fall. Hausfrau, 37jährig. IV para, hereditär nicht belastet. Verschlimmerung der tuberculösen Lungenaffection II. Stadiums durch die bestehende Schwangerschaft. Complicationen: Erbrechen, Pleuritis serosa. Einleitung des künstlichen Abortes im VI. Monat. Besserung im Wochenbett. Weiterer Verlauf: Andauernde Besserung, Stillstand der Lungentuberculose, Arbeitsfähigkeit, also positiver Erfolg laut Bericht des Arztes, Dr. Staub, vom 25. 11. 1905: „Nach der Geburt dauernde Besserung. Positiver Erfolg. Bei der Entlassung II. Stadium (Turban)." Ausgang laut obigem Bericht: "Bis heute voll leistungsfăhig geblieben" - 3 Jahre 6 Monate nach" dem künstlichen Abort. Patientin befand sich vor und nach der künstlichen Entbindung zur Kur im Sanatorium Wald.

19. Fall. Hausfrau, 22jährig. I para, hereditär nicht belastet. Verschlimmerung der tuberculösen Lungenaffection I. Stadiums durch die bestehende Schwangerschaft. Complication: Knochen- und Hauttuberculose. Einleitung. des künstlichen Abortes im IV. Monat. Zustand im Wochenbett zunächst unverändert, dann Verschlimmerung. Weiterer Verlauf: Andanernde Verschlimmerung, Fortschreiten der Lungentuber- 
culose, Arbeitsunfähigkeit, Tod, also negativer Erfolg laut Bericht der Aerzte, Dr. Arnold und Dr. Henschen (im Auftrage von Herrn Prof. Krönlein), vom 23. 12. 1905 und 6. 1. 1906. Ausgang laut obigen Berichten: Ausser der Lungentuberculose machte namentlich die Hautund Knochentuberculose rapide Fortschritte, "Bei Frau S. trat die Lungentuberculose gegen die Hautaffection nach und nach fast ganz in den Hintergrund" schreibt Dr. A rnold. Die Patientin erlag ihrem Leiden am 22. 7. 1903 - 1 Jahr 2 Monate nach der küustlichen Entbindung.

Das vorliegende Krankenmaterial giebt uns Anlass zu folgenden Ausführungen:

Im Zeitraum vom 11. Juli 1893 bis 17. Mai 1902 wurde auf der Züricher Frauenklinik auf die ausdrüękliche Indication Lungentuberculose hin die Schwangerschaft bei 22 Frauen künstlich unterbrochen. Nicht berücksichtigt wurde, weil für die nachfolgende Besprechung ausser Betracht kommend, ein Fall, über welchen wir weiter unten berichten werden. Nicht verwendet wurden von den obigen 22 Fällen 3, in welchen ein Bericht über den weiteren Verlauf und Ausgang nicht erhältlich war.

Von den 19 in Betracht kommenden Frauen standen im Alter von 20-30 Jahren 12 oder 63 pCt., 7 oder 37 pCt. vertheilten sich auf das vierte und die erste Hälfte des fünften Dezenniums. In die erste Verpflegungsklasse der Klinik liessen sich 8 , in die zweite 10 Patientinnen aufnehmen, 1 wurde ausserhalb der Klinik behandelt. Die Kranken der ersten Kategorie befanden sich fast durchweg in guten, zwei derselben (Fall 11 und 13) in vorzüglichen Lebensverhältnissen. Die Franen der zweiten Klasse mussten fast ohne Ausnahme nach der Entlassung aus der Klinik wieder ihre Haus- und Berufsarbeit aufnehmen.

Hereditäre Belastung war 11 Mal vorhanden, 8 Mal fehlte sie. Von den verstorbenen. Frauen waren drei belastet, zwei nicht.

Es waren der Schwangerschaftszahl nach 3 Erstgebärende, 3 II para, 4 III p., 6 IV p., $2 \mathrm{~V}$ p., 1 XI p.

In 17 von 19 Fällen, also 90 pCt. derselben, manifestirte der Einfluss der Schwangerschaft sich in einer Verschlimmerung des Zustandes, in $10 \mathrm{pCt}$. oder $2 \mathrm{Mal}$ war ein ungünstiger Einfluss, wenigstens objectiv, nicht nachweisbar.

Den vorhandenen Befunden nach waren 9 Kranke in das erste, 6 in das zweite, 4 in das dritte Stadium nach Turban einzureihen.

Die Lungentuberculose war complicirt in 9 Fällen und zwar je $1 \mathrm{Mal}$ mit schwerer Haut- und Knochentuberculose, hochgradiger 
Anämie, Kehlkopftuberculose und Coxitis, Pleuritis serosa und Brechen, Pleuritis serosa mit Brechen und Diarrhoen, je 2 Mal mit Brechen, Kehlkopftuberculose. Vier der complicirten Fälle $=44$ pCt. gehören unter die negativen, $5=56 \mathrm{pCt}$. unter die positiven Erfolge und zwar entfallen auf das erste und zweite Stadium je 2 positive und 1 negativer Erfolg bezw. Todesfall, auf das dritte ein positiver und 2 negative Resultate oder Sterbefälle.

Von den 5 im weiteren Verlaufe erfolgten Todesfällen war die Lungentuberculose complicirt in $4=4 / 5$ derselben.

Nur 1 Mal wurde die künstliche Frühgeburt im zehnten Monat, $18 \mathrm{Mal}$ der künstliche Abort eingeleitet und zwar $1 \mathrm{Mal}$ im zweiten, $6 \mathrm{Mal}$ im dritten, $5 \mathrm{Mal}$ im vierten, $1 \mathrm{Mal}$ im vierten bis fünften, $2 \mathrm{Mal}$ im fünften, $1 \mathrm{Mal}$ im fünften bis sechsten, $1 \mathrm{Mal}$ im sechsten, 1 Mal im siebenten Monat.

Die Narkose wurde bei 15 Frauen angewandt und von allen ohne die geringsten unangenehmen oder schädlichen Erscheinungen oder Folgen ertragen.

Bei einer Frau, welche schon vor der Operation blutigen Ausworf hatte, wurde auch während der Geburt 2 Mal dunkler blutig gefärbter Auswurf beobachtet, im übrigen wurde die Lungentuberculose durch den Eingriff selbst in keiner Weise nachweisbar ungünstig beeinflusst.

Im Wochenbett trat eine Besserung der Lungentuberculose ein bei $15=80$ pCt. derWöchnerinnen, die während der Schwangerschaft bestehende Verschlimmerung blieb auch im Puerperium fortbestehen bei $2=10$ pCt., bei den 2 Frauen oder in wejteren 10 pCt., bei welchen während der Gravidität ein ungünstiger Einfluss nicht zu constatiren war, blieb der Zustand der Lungen unverändert.

Ueber den weiteren Verlauf ergaben sich folgende Resultate: positiv war der Erfolg in $14=74$ pCt., negativ in $5=26$ pCt. der Krankheitsfälle, wenn wir das Stadium nicht in Betracht ziehen.

Mit Berücksichtigung desselben ändern die Resultate sich in folgender Weise: von 9 Fällen des I. Stadiums war der Erfolg positiv in $8=89$ pCt., negativ in $1=11$ pCt.; von 6 Fällen des II. Stadiums positiv in $5=83$ pCt., negativ in $1=17$ pCt.; von 4 Fällen des' III. Stadiums positiv in $1=25$ pCt., negativ in $3=75$ pCt.

Interessant ist ein Vergleich unserer Resultate mit den Ergebnissen von Statistiken, welche Aufschluss über die Erfolge der Therapie bei Lungentuberculose im Allgemeinen geben. Wir greifen 
die Turban'sche Zusammenstellung heraus, deren Material wir grossentheils mitbeobachtet haben. Der Entlassungserfolg im Sinne von Brebmer's sogenannter absoluter und relativer Heilung betrug nach einer durchschnittlich $7 \frac{1}{2}$ monatlichen Kurdauer für das I. Stadium 97,9 pCt. ( 89 pCt.), für das II. 73,2 pCt. (83 pCt.), für das JII. 23,6 pCt. (25 pCt.). Die Zahlen in Parenthese bedeuten unsere Resultate, die bei der Berücksichtigung des künstlichen Abortes allein sich noch verbessern würden.

Rechnen wir die bei Fall 2 und 4 mit positivem Erfolge auf der Züricher Klinik ausgeführten künstlichen Abortoperationen der Jahre 1894 und 1896 dazu, so verbessern die Ergebnisse der Fälle des I. Stadiums sich auf folgende Weise: von 11 Operationen hatten positiven Erfolg $10=91$ pCt., negativen $1=9$ pCt.

Im weiteren Verlaufe starben 5 Frauen und zwar nach folgenden Zeiträumen vom Datum der Entbindung an gerechnet: nach 5 Wochen eine, nach 6 Monaten zwei, nach 1 Jahr 2 Monaten eine, nach 2 Jahren 9 Monaten eine. Bei vier der Verstorbenen war die Lungentuberculose zum Theil schwer complicirt. Die Zahl der negativen Erfolge bezeichnen zugleich die Mortalitätsziffern.

Der schliessliche Ausgang bei den 14 Fällen mit positivem Resultate war folgender: es war die Lungentuberculose der betreffenden Frauen noch stationär, bezw. ausgeheilt und es waren arbeits- oder leistungsfähig nach Ablauf eines Zeitraumes von:

\begin{tabular}{|c|c|c|c|c|c|c|c|}
\hline 12 & iren, & 5 & Monaten & nach & der & künstlichen & Entbindung \\
\hline 12 & $n$ & 3 & $"$ & $n$ & $n$ & $n$ & $n$ \\
\hline 10 & $n$ & 11 & $n$ & $n$ & $n$ & $n$ & $n$ \\
\hline 10 & $n$ & 7 & $n$ & $n$ & $n$ & $n$ & $n$ \\
\hline 9 & $n$ & 9 & $n$ & $n$ & $n$ & $n$ & $n$ \\
\hline 8 & $n$ & 1 & $n$ & $n$ & $n$ & $n$ & $n$ \\
\hline 6 & $\eta$ & 9 & $n$ & $n$ & $n$ & $n$ & $n$ \\
\hline 3 & $"$ & 9 & $n$ & $n$ & $n$ & $n$ & $"$ \\
\hline 3 & $n$ & 6 & $n$ & $n$ & $n$ & $n$ & $n$ \\
\hline 3 & $"$ & 1 & $n$ & $"$ & $n$ & $n$ & $n$ \\
\hline 2 & $n$ & 11 & $n$ & $"$ & $n$ & $n$ & $n$ \\
\hline & & 6 & $n$ & $n$ & $n$ & $n$ & $n$ \\
\hline & & 2 & $n$ & $n$ & $\Rightarrow$ & $n$ & $n$ \\
\hline
\end{tabular}

Nach Abzug der einen künstlichen Frühgeburt ergeben sich für den künstlichen Abort folgende Resultate bei Nichtberücksichtigung des Stadiums: positiver Erfolg in $14=78$ pCt., negativer 
in $4=22$ pCt. der Fälle, bei Berücksichtigung desselben bleibt das Ergebniss für das erste und zweite dasselbe. Bei den drei Frauen des dritten, welche dann verbleiben, wurde erzielt ein positiver Erfolg in $1=33 \frac{1}{2}$ pCt, ein negativer ergab sich bei $2=$ $66^{2} \frac{1}{2}$ pCt. der Fälle.

Die Indication wurde $2 \mathrm{Mal}$ in rein prophylaktischem Sinne gestellt, weil im Verlaufe früherer Schwangerschaften und Geburten spontaner Natur die Lungentuberculose sich verschlimmert hatte, 17 Mal, weil im Laufe der in Frage kommenden Gravidität eine Verschlimmerung aufgetreten war.

Die Operation selbst, fast durchwegs in Dilatation nach Hegar, Tamponade und manneller Ausräumung bestehend, verlief sowohl in den $19 \mathrm{im}$ Vorhergehenden besprochenen, als auch in den 3 wegen mangelnder Berichte über den weiteren Verlauf und Ausgang nicht verwendeten Fällen glatt und ohne Störung.

In dem einen Falle, welcher, wie oben bemerkt, nicht berücksichtigt wurde, sollte im dritten Monat der Schwangerschaft der Abort eingeleitet werden. Durch die Ungeschicklichkeit eines die Operation ausführenden Assistenten war eine Verletzung gesetzt worden, welche die Totalexstirpation des septischen Inhalt enthaltenden Uterus nöthig machte. Die Patientin starb 3 Tage später. an septischer Peritonitis.

Die künstliche Unterbrechung der Schwangerschaft war in allen Fällen durch einen oder mehrere Aerzte bejahend begutachtet worden.

Die aus dem vorliegenden Material der Züricher UniversitätsFrauenklinik sich ergebenden Jehren liegen, wie wir eingangs dieser Arbeit bemerkten, den zum Theil bekannten zum Theil auf Grund des Gesammtmaterials von uns aufgestellten Erfahrungs- und Schlusssätzen bereits mit zu Grunde.

Den Abschluss auch dieser Arbeit möge der Versuch bilden, dem vor eine Entscheidung gestellten Arzte für seine Entschliessung und sein Handeln eine kurze Wegleitung an die Hand zu geben.

Als prophylaktische Maassnahme kommt das Eheverbot in Betracht. Ein absolutes Eheverbot würden wir, auch wenn es practisch durchführbar wäre, für unberechtigt einem grossen Theil von Mädchen und Frauen gegenüber erachten, dagegen halten wir eine Einschränkung des Eheconsenses für nothwendig und gerechtfertigt. Er ist Patienten mit activer und auch schwerer stationärer Lungentuberculose zu verweigern, in den übrigen Fällen soll er bei 
ausgesprochener Erkrankung dann erst ertheilt werden, wenn die Krankheit mindestens 1-2 Jahre nachgewiesenermaassen stationär geblieben war. Es besteht unseres Erachtens kein berechtigender Grund, weibliche aus belasteter Familie stammende oder latenter Tuberculose blos verdächtige Personen von den Freuden des Mutterglückes und des Familienlebens von vornherein auszuschliessen. Ausser dem Stadium und Charakter der Krankheit und den schon erwähnten in Betracht kommenden Factoren ist besonders die sociale Stellung der Ehestandscandidaten zu berücksichtigen in dem Sinne, als bei Wohlhabenden der Consens eher ertheilt werden darf als bei weniger oder unbemittelten Kranken.

Wo immer der Arzt dazu Gelegenheit hat, soll er die Ehestandscandidaten und deren Angehörige in sachlicher klarer Weise über die möglichen Gefahren und Folgen für den Patienten selbst. und dessen Nachkommenschaft aufklären und belehren, im Uebrigen aber die freie Entscheidung den Betheiligten überlassen, welche die Verantwortlichkeit für ihr Handeln dann selbst zu tragen haben.

Ist man der lungentuberculösen Gravida gegenüber vor eine Entscheidung für sein therapeutisches Handeln gestellt, so muss vor Allem festgestellt werden, ob es sich um progressive oder stationäre Krankheitsprocesse handelt.

Ist durch eine gesicherte Diagnose festgestellt, dass eine während der Schwangerschaft frisch oder recidivirend auftretende Lungentuberculose progressivgeworden ist, so muss die erste Aufgabe des Arztes darin bestehen, die Krankheit vorerst durch erfahrungsgemäss wirksame Mittel zu bekämpfen und zum Stillstand zu bringen.

Dringende Fälle ausgenommen, bezeichnen wir den Versuch, die Krankheit durch rationelle antituberculöse Behandlung zum Stillstand zu bringen, als unerlässliche Vorbedingung für jedes operative Vorgehen. Es kann nicht. unsere Aufgabe sein, hier eine Abhandlung über rationelle Phthiseotherapie zu schreiben, darauf müssen wir aber hinweisen, dass gerade solche therapeutische Maassnahmen, welche als besonders wirksame Kampfmittel gegen die Krankheit empfohlen werden, den schwangeren Frauen gar nicht oder nur sehr schwer zugänglich sind und za Gute kommen, wir meinen die Unterbringung solcher Kranken in Heilstätten.

Bei der Errichtung von Lungenheilstätten sollte auch auf diese Kategorie von Kranken Rücksicht genommen werden. Auch die Archiv für Gynäkologie. Bd. 83. H. 2. 
möglichst frühe Verbringung auf besondere Abtheilungen der Gebärhäuser und die Ueberweisung an und Behandlung von Fürsorgestellen aus würde sicherlich schon erfreuliche Erfolge erzielen.

Durch ein derartiges abwartendes Verhalten in Verbindung mit einer rationellen Behandlung werden ohne Zweifel manche positive Erfolge erreicht werden, doch wird dasselbe fraglos in anderen Fällen unwirksam bleiben und nun tritt die Frage der Indicationsstellung zur künstlichen Unterbrechung der Schwangerschaft an den Arzt heran. Auf die Selbsthülfe der Natur sich zu verlassen, empfiehlt sich deshalb nicht, weil erfahrungsgemäss der spontane Abort selten eintritt und die Fälle von spontaner Frühgeburt, welche fast durchweg nur bei schwerer Erkrankung beobachtet sind, meist einen sehr ungünstigen Verlauf nehmen.

Der Arzt wird zur Entscheidung gedrängt. In welchen Fällen soll nun zum operativen Eingriff geschritten werden?

Allgemein gesprochen erachten wir die künstliche Unterbrechung der Schwangerschaft bei Lungentuberculose für indicirt in Fällen, wo die Prognosestellung dës mütterlichen Leidens einen Stillstand, eine Ausheilung erwarten lässt oder, bei aussichtsloser Erkrankung der Nutter, die Rettung des Kindes in Frage kommt.

Für die Prognosestellung des mütterlichen Leidens sind die Erfahrungen der Tuberculoseforschung im Sinne der im Vorhergehenden niedergelegten Erfahrungssätze, für die Prognose des Kindes diejenigen des Geburtshelfers maassgebend.

Wir warnen aber ausdrücklich vor einer schematischen Anwendung der Stadieneintheilung bei der Indicationsstellung.

Auf Einzel-Indicationen eingehend, halten wir die künstliche Entbindung für indicirt in Fällen, in welchen durch eine conservative Therapie eine während der Schwangerschaft progressiv gewordene Lungentuberculose nicht zum Stillstand und zur Ausheilung gebracht wird. Ferner dann, wenn äussere Umstände eine solche Behandlungsmethode unmöglich machen, was am häufigsten wohl bei wenig oder unbemittelten Kranken zutreffen wird.

Der operative Eingriff bezweckt in erster Linie die Beseitigung der schädigenden Ursache und soll dadurch einen Stillstand und eine Ausheilung in die Wege leiten. 
Wir bezeichnen diese Art der Indicationsstellung als therapeutische.

Wie soll sich der Arzt einer Gravida mit stationärer Lungentuberculose gegenüber verhalten? Er wird in solchen Fällen im Allgemeinen keinen Grund haben, aus seiner abwartenden Stellung herauszutreten. Wo und wie immer, thunlich, wird er einem Activ- und Progressivwerden der Krankheit vorbeugen, ihm prophylaktisch entgegenarbeiten.

Schwieriger gestaltet sich die Frage, wenn aus dem Verlaufe vorhergegangener Schwangersehaften hervorgeht, dass bei normalem Verhalten der Gravidität nach erfolgter spontaner Geburt eine Lungentuberculose activ oder progressiv wurde.

Wenn in derartigen Fällen die Gesundheit der Mutter, welche schon durch vorhergegangene Geburten eine Schädigung erlitten hat und, obwohl Rückschlüsse vom Verlaufe vorhergegangener auf denjenigen künftiger Entbindungen nicht ohne Weiteres zutreffend sind, durch eine kommende aufs Neue bedroht erscheint, so dass bei ähnlichem Verlaufe eine Wiederherstèllung aller Wahrscheinlichkeit nach nicht mehr möglich sein wird, sollte unter solchen Umständen der Arzt nicht berechtigt sein, die Schwangerschaft zu unterbrechen in dem Bestreben, den so grosse Anforderungen an die Kranke stellenden rechtzeitigen Geburtsact nicht abzuwarten und den leichteren Eingriff der künstlichen Fehlgeburt im Interesse der Mutter an ihr vorzunehmen?

Wenn in dergleichen Fällen die nach der Entbindung eingetretene Verschlimmerung wirklich den Generationsvorgängen, nicht etwa anderen schädigenden Momenten zuzuschreiben war, möchten wir die Frage bejahend beantworten, aber nur in diesem Falle.

Wir nennen diese Indicationssteliung prophylaktische.

Wurde auch durch eine derartige Intervention in prophylaktischem Sinne ein positiver Erfolg nicht erreicht, so dürfte bei solchen Franen, wie auch bei denjenigen, bei welchen eine künstliche Entbindung wegen progressiver Lungentuberculose ungünstige Resultate ergab, die Ausführung der künstlichen Sterilisation am Platze sein.

Wann soll im gegebenen Falle die künstliche Entbindung eingeleitet werden? 
Auch die Wahl des Zeitpunktes der Intervention muss von der Prognosestellung für Mutter und Kind abhängig gemacht werden. Wir haben oben schon zur Prognosestellung bei der Lungentuberculose schwangerer. Frauen in Form eines Erfahrungssatzes Stellung genommen. Wir müssen hier Folgendes ergänzend beifügen.

Diese Prognosestellung begegnet ganz besonderen Schwierigkeiten in Bezug auf die einzuschlagende Therapie, bezw. auf die Wahl des Zeitpunktes eines als nothwendig erachteten Eingriffes, da der Arzt vom Momente der Conception an verpflichtet ist, auch das Interesse des Productes derselben und zwar nach der Auffassung vieler Aerzte in demselben Maasse wie das der Mutter zu wahren.

Es ist nun aber nicht zu bestreiten, dass, wenn eine Einwirkung anderer Factoren als der Schwangerschaft allein auszuschliessen ist und mit dem Fortschreiten derselben auch eine frisch entstandene oder recidivirende Lungentuberculose progressiv wird, die Entstehung und Entwicklung des Fötus allein als Ursache dieser Verschlimmerung anzusehen ist. Dadurch entsteht naturgemäss ein Antagonismus der mütterlichen und kindlichen Interessen, welcher es dem Arzte je nach der Phase der Schwangerschaft geradezu unmöglich macht, seine therapeutischen Maassnahmen so einzurichten, dass durch dieselben in gleicher Weise das Wohl beider Schutzbefohlenen berücksichtigt: wird.

Wir glauben, dass der Arzt principiell auf den Standpunkt sich stellen soll, im Interesse von Mutter und Kind zu handeln. In der Praxis wird er aber oft genug in die Lage kommen, denselben verlassen und sich entscheiden zu müssen, vorwiegend das Wohl und Wehe eines der beiden zur Richtschnur seines Handelns zu nehmen. Bestimmend für den Geburtshelfer wird in der Regel wohl der Zeitpunkt der Lebensfähigkeit des Kindes sein, während der innere Arzt die Prognose des mütterlichen Leidens vornehmlich seinen Entschliessungen zu Grunde legen wird.

Möge der letztere bedenken, dass jede Spanne Zeit des Zuwartens der Lebensfähigkeit des Kindes zu Gute kommt, vergesse aber auch der erstere nicht, dass jeder Aufschub das Leiden der Mutter und damit die Aussicht auf die Wiederherstellung und Erhaltung ihrer Gesundheit in Frage stellt.

Es besteht eben ein wesentlicher Unterschied im Verlaufe anderer pathologischer Zustände, welche als be- 
rechtigte Indicationen zur künstlichen Unterbrechung der Schwangerschaft anerkannt werden, und der Lungentuberculose, da die einmal bei dieser in den Lungen gesetzten Läsionen nicht vorübergehender Natur sind sondern je nach dem Grade der Verschlimmerung eine geringere oder erheblichere Schädigung der Gesundheit und des Lebens der Mutter zur Folge haben, auch im günstigsten Falle, das heisst, wenn nach der Entbindung ein Stillstand und eine Ausheilung eintritt, während bei manchen jener anderen Krankheitszustände die Frauen nach der Geburt sich. ihrer früheren Gesundheit und Leistungsfähigkeit erfreuen.

Wir haben diese Thatsache in der gesammten uns vorliegenden Literatur, soweit uns erinnerlich, nirgends erwähnt gefunden.

Wir suchten dem Standpunkt des Geburtshelfers und internen Arztes gerecht zu werden, wenn wir bezüglich der Wahl des Zeitpunktes der Intervention folgende Sätze aufstellteń:

Im Interesse der Mutter erscheint uns die künstliche Unterbrechung der Gravidität angezeigt und zwar so frühzeitig wie möglich, wenn die Progressivität der Lungentuberculose auftritt oder andauert zu einer Zeit, wo die Frucht noch nicht lebensfähig ist und nach ärztlichem Ermessen durch ein Abwarten bis zum Zeitpunkt der Lebensfähigkeit die Prognose der Mutter in der Weise sich verschlechtern wird; dass ein positiver Erfolg in unserem Sinne nicht mehr zu erwarten ist.

Schematisch gesprochen, und das gilt für jede Indicationsstellung, bei welcher das Interesse der Mutter allein oder vorwiegend berücksichtigt werden soll, muss der Arzt verhindern, dass in Folge Zuwartens die Krankheit vom ersten ins zweite, vom zweiten in das dritte Stadium übertrete (Fall 7).

Ist zur Zeit der Progressivität der Krankheit das Kind lebensfähig, so hat die Indicationsstellung auch das Interesse des Kindes zu wahren, sicherlich eine schwere Aufgabe, da einerseits das Wohl der Mutter meist die sofortige Intervention verlangt, dasjenige des Kindes ein möglichstes Hinausschieben des Zeitpunktes wünschbar macht. Das sind diejenigen Fälle, in welchen der Arzt in dem Bestreben, ein möglichst lebensfähiges Kind zu erhalten, leicht Gefahr läuft, den für die Mutter. 
günstigsten Moment der Operation zu verpassen. Wir möchten der Indicationsstellung in solchen Fällen folgende Fassung geben:

Nach erreichter Lebensfähigkeit des Kindes ist die Schwangerschaft bei fortschreitender Lungentuberculose der Mutter sofort zu unterbrechen, sobald nach ärztlichem Ermessen zu befürchten steht, dass bei weiterem Zuwarten die Prognose der Mutter sich so verschlimmern wird, dass ein positiver Erfolg nicht mehr zu erwarten ist.

Ganz besonders schwierig wird die Entscheidung für den Arzt, wenn die Verschlimmerung des mütterlichen Leidens dem Zeitpunkt nahe liegt, welcher eine Lebensfähigkeit des Kindes in Bälde erwarten lässt. Unter thunlichster Berücksichtigung der Prognose darf der Arzt bei solcher Lage des Falles die Verantwortung auf sich nehmen, den Zeitpunkt zu Gunsten des Kindes so weit als nothwedig hinauszuschieben.

Das Interesse des Kindes allein wird bei prognostisch aussichtsloser Erkrankung der Mutter in Frage kommen. Leitend für die Wahl des Zeitpunktes wird unter diesen Umständen der Gedanke und das Bestreben sein, bei möglichster Berücksichtigung und Schonung. der Mutter ein möglichst lebensfähiges Kind zu erhalten.

Der Vorschlag verschiedener Autoren, die Schwangerschaft bei Lungentuberculose quälender Beschwerden wegen im Sinne einer rein symptomatischen Indicationsstellung "künstlich zu unterbrechen, dürfte denn doch sehr schwere Bedenken erregen.

Indem. wir nochmals vor schematischer Indicationsstellung warnen, stellen auch wir den Grundsatz auf: Die Indication muss ausnahmslos von. Fall zu. Fall gestellt werden.

Es gehört ausser einer gewissenhaften Leberwachung und Beobachtung der Kranken sicherlich kein geringer Grad von Erfahrung: auf dem Gebieté der Lungentuberculose und Geburtshälfe zu einer relativ sicheren Prognosestellung. für Mutter und Kind und kein Arzt, dem sein eigenes. Wissen und Können eine befriedigende Antwort auf die Frage der Indicationsstellung nicht ertheilt, wird sich etwas vergeben, wenn er sich bei erfahrenen Kollegen Auskunft und Rath holt.

Wenn wir von der Wahl der Operationsmethode sprechen sollen, so ist diejenige die empfehlenswertheste, welche, die Kranke am meisten schonend, zum Ziele führt. 
Pradella, Künstliche Unterbrechung der Schwangerschaft. 407

Die Narkose wird nach unseren Erfahrungen von den gebäronden tuberculösen Frauen gut vertragen.

Nach erfolgter Entbindung, sei diese spontan oder künstlich, müssen die tuberculösen Wöchnerinnen so lange in Beobachtung und, wo eine solche sich als nothwendig herausstellt, rationeller antituberculöser Behandlung stehen, bis ein endgültiges Urtheil über den weiterem Verlauf und definitiven Ausgang möglich ist. Nur auf diese Weise wird der Einfluss von Geburt und Wochenbett auf die Lungentuberculose in objectiver Weise mit Sicherheit festgestellt werden können.

Als selbstverständliche Anordnung betrachten wir das Stillverbot bei tuberculösen Wöchnerinnen.

Eine ausserordentlich wichtige prophylaktische Maassnahme nach der Entbindung betrifft die Conceptionsverhinderung bei tuberculösen Frauen.

Eine neue Conception sollte bei Frauen, bei welchen ein ungünstiger Einfluss im Verlaufe der Generationsvorgänge auf die Lungentuberculose sich gezeigt hatte, vor Ablauf von mindestens $1-2$ Jahren vom Datum des Stillstandes der Erkrankung ab, nicht stattfinden. Es liegt auf der Hand, welch' eminente Bedeutung der Conceptionsverhinderung, welcher leider viel zu wenig Beachtung und Werth beigemessen wird, zukommt und der Arzt wird sich ausserordentliche Verdienste erwerben; welcher keine Mühe schent, die Betheiligten in geeigneter Weise zu belehren und zu instruiren.

Durch unsere bisherigen Ausführungen erachten wir uns der Nothwendigkeit enthoben, die Berechtigung zur Conceptionsverhinderung noch näher zu begründen.

Die Berechtigung zur künstlichen Unterbrechung der Schwangerschaft bei Lungentuberculose vom medicinischen Standpunkte ans erachten wir durch die Erbringung des Nachweises, dass durch dieselbe positive Erfolge erzielt werden, für ausser Frage gestellt.

Hinsichtlich der Bedenken dem rechtlichen Standpunkte gegenüber sind wir der Meinung, dass bei der Unmöglichkeit einer allgemeinen präcisen Indicationsstellung gesetzgeberische Bestjmmungen die Lage des Arztes nur noch unsicherer gestalten würden.

Wir glauben einen Arzt, welcher nachfolgende Vorsichts,maassregeln beobachtet, dem Gesetze und Publicum gegenüber 
als wirksam geschützt und empfehlen deren Beobachtung auch den öffentlichen und Privatanstalten:

Sicherung der Diagnose der Lungentuberculose, bezw. der Prógressivität derselben auf Grund genau geführter Krankengeschichten, Zuziehung eines oder mehrerersachkundiger Aerzte zur Bestätigung der Diagnose und Indication des Eingrifis, schriftliche Einwilligung der Mutter und des Gatten oder sonst verantwortlicher Personen wach gründlicher, sachlicher Auseinandersetzung der Sachlage mit Ueberlassung der persönlichen freien Entscheidung in jedem Falle, Ausstellung eines gegenseitigen schriftlichen Gutachtens zwischen dem consultirten und consultirenden Arzte.

Da es wohl ausgeschlossen ist, dass eine stationäre oder fortschreitende Lungentuberculose einem genau und gewissenhaft beobachtenden Arzte, noch viel weniger zweien oder mehreren ertolgreich vorgetäuscht werden kann, so ist einem Missbrauch seitens der Patientin unschwer vorzubeugen. Dem Wunsche einer Kranken selbst nach Intervention wird der Arzt nur Folge geben, wenn er es vor seinem eigenen Wissen und Gewissen verantworten kann.

Wir schliessen die Arbeit, indem wir der Ueberzeugung Ausdruck geben, dass durch ein zielbewusstes Zusammenarbeiten der Geburtshilfe und inneren Medizin mit der Zeit auf Grund eines quantitativ und qualitativ geeigneten Materials die Indication noch klarer und präciser $\mathrm{zu}$ stellen sein wird und eine möglichst sichere Indicationsstellung bedeutet den Schlüssel zur Lösung der Streitfrage.

\section{Lit eratur.}

1. Barnes, R., The indications for the induction of permature labor. The Lancet. 11. August. 1894.

2. Berliner klinische Wochenschrift. Vierter internationaler Congress für Geburtshülfe und Gynäkologie in Rom. Special-Referat von Dr. L. Pulvermacher. No. 39. S. 926.1902.

3. Charpentier, A., Traité pratique des accouchements. Tome 1. p. 584. (Baillières.) Paris. 1883.

4. Duncan, William, Should pregnanoy be terminated prematurely in cases of phthisis? Transact. of the obstetr. Soc. of London. Vol. $32 . \quad$ p. 7. 1890. (Mit Discussion.)

5. Eich, Gottfried, Zur Frage des künstlichen Abortes bei Phthisis pulmonum. Inaug.-Diss. Marburg. 1904. 
6. Fellner, 0., Tuberculose und Schwangerschaft. Wiener med. Wochensohrift. No. 25-27. S. 1158 etc. 1904.

7. Freund, W. A., Die künstliche Unterbiechung der Schwangerschaft, ihre Indicationen und ihre Methodik. Die deutsche Klinik am Eing. des 20. Jahrhunderts. S. 169. 1901.

8. Fritsch, H., Geburtshülfe. Leipzig. (Hirzel.) 1904. - Der künstliche Abort. Deutsche med. Wochenschr. No. 48. S. 1759.1904.

9. Funke, Indicationen des artificiellen Abortes bei Phthisis pulmonum, Unterelsässisch. Aerztev. Bericht. Münchener med. Wochensch. S. 44. 1904. (Mit Discussion.)

10. Gallatia, E., Einleitung der künstlichen Frühgeburt bezw. des Abortes in den Jahren 1898 bis 1. Juli 1904. Aus d. gyn.-geb. Abtheil. des Prof. Dr. Alfred v. Valenta in Laibach. Wiener klin. Wochenschr. No. 45. S. 1212. 1904.

11. Gerhardt, Ueber Eheschliessung Tuberculöser. Deutsche med. Wochenschrift. Vereinsbeilage. S. 177. 1900. (Mit Disoussion.) Ausführlich in der Zeitschr. f. Tuberculose und Heilstätten.

12. Hahn, W., Tuberculose und Schwangerschaft. Berliner klin. Wochenschr. No. 52. 1903:

13. Hamburger, C., Ueber die Berechtigung und Nothwendigkeit bei taberculösen Arbeiterfrauen die Schwangerschaft zu unterbrechen. Berlin. klin. Wochenschr. No. 45 eto. Discussion hierzu. No. 25. S. 591. 1902.

14. Hantke, R., Die medicinischen Indicationen zur Unterbrechung der Schwangerschaft. Sammelbericht. Monatsschr. f. Geb. u. Gyn. Bd. 16. S. 370. 1902.

15. Jacoby, Ueber die künstliche Unterbrechung der Schwangerschaft durch die Bougiemethode. Erfahrungen über Indication und Methode aus 228 Fällen. Dieses Archiv. Bd. 74. H. 2. 1904.

16. Kaminer, Siegfried, Ueber den Einfluss von Schwangerschaft und Entbindung auf den phthisischen Process und den therapentischen Werth der Einleitung von künstlichen Aborten. Deutsche med. Wochenschr. No.35. S. 587. 1901. Discussion hierzu. No. 26. S. 197 u. No. 27. S. 212. Vereinsbeilage. 1901.

17. Kellmann, Fr., Zur Frage der künstlichen Unterbrechung der Schwangerschaft wegen Lungentuberculose. Inaug.-Diss. Berlin. 1905.

18. Kikuth, M., Ehe, Tubereulose und Schwangerschaft. St. Petersburger med. Wochenschr. No. 50. S. 549. 1904.

19. Kossmann, R., Das Recht des Arztes und der Mutter über. Leben und Tod des ungeborenen Kindes. Die Heilkunde. Heft 3. S, 20. 1902. Discussion hiorzu. Berliner klin. Wochenschr. No. 7. S. 149. 1902.

20. Leopold, G., Ueber die künstliche Frühgeburt bei hoffnungsloser Erkrankung der Mutter. Dieses Arch. Bd. 14. S. 299. 1879. Discussion hierzu. S. 313.

21. Lomer, Ueber die Indicationen zum künstlichen Abort. Centralbl. f. Gyn. No. 45. S. 1138, 1141 u. 1173. 1894. (Mit Discussion.)

22. Lop, P.A., D $\ominus$ l'avortement et de l'accouchement prématuré dans la tuberculose pulmonaire. Arch. de toc. et de gyn. Tome 21. p. 575. 1894. 
(Mit Referat über den Congress der italienischen Geburtshelfer in Rom 1886.)

23. Merletti, C., Tuberculosi e gravidanza. Arch. ital. di Ginec. Vol. 2. No. 4. 1904. (Mit Discussion.)

24. Mübsam, A., Ueber Indicationen zur Einleitung des Aborts. Inaug.-Diss. Berlin. 1904.

25. Pförtner, Otto, Ueber die Unterbrechung der Schwangerschaft bei tuberculösen Frauen. Inaug.-Diss. Berlin. 1904.

26. Proust, Gabr., Influence qu'excercent la grossesse, l'accouchement et l'état puérpéral sur la tuberculose pulmonaire. Thèse de Paris. 1903.

27. Rebière, J. J., Contribution à l'étude de la tuberculose dans ses relatións avec la grossesse et les suites des couches. Thèse de Paris. 1900.

28. Ruge, Paul, Ueber die künstliche Unterbrechung der Schwangerschaft wegen starkem Erbrechen und Schwindsucht. Berliner klin. Wochenschr. No. 33. S. 1035 . 1905.

29. Schauta, F., Die Einleitung der Geburt wegen innerer Erkrankungen. Monatssebr. f. Geb. u. Gyn. Bd. 16. S. 470. 1902.

30. Towsend, Ch. W., Phthisis and Child-Bearing. Boston med. and surg. journ. p. 391, 398. 1897. (Mit Discussion.)

31. Warren, Ed., Does pregnancy accelerate or retard the development of tubercles of the lungs in persons predisposed to this disease? The americ. journ. of the med: seience Vol. 34. p. 87. 1857. (Mit Discussion.)

32. v. Winckel, F., Handbuch der Geburtshülfe. Bd. II. H. 1. S: 589. (H. W. Freund) und Bd. II. H. 2. S. 1303. (L. Seitz.) 